ARTICLE

https://doi.org/10.1038/s41467-019-11918-y

\title{
Discovery and preclinical evaluation of anti-miR-17 oligonucleotide RGLS4326 for the treatment of polycystic kidney disease
}

Edmund C. Lee ${ }^{1}$, Tania Valencia', Charles Allerson', Annelie Schairer ${ }^{1}$, Andrea Flaten², Matanel Yheskel ${ }^{2}$,

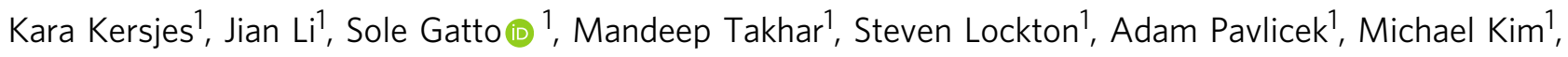

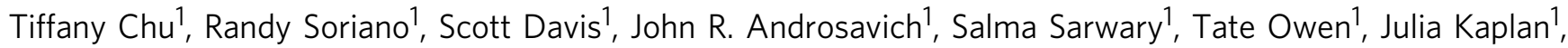
Kai Liu', Graham Jang ${ }^{1}$, Steven Neben¹, Philip Bentley ${ }^{1}$, Timothy Wright ${ }^{1} \&$ Vishal Patel ${ }^{2}{ }^{2}$

Autosomal dominant polycystic kidney disease (ADPKD), caused by mutations in either PKD1 or PKD2 genes, is one of the most common human monogenetic disorders and the leading genetic cause of end-stage renal disease. Unfortunately, treatment options for ADPKD are limited. Here we report the discovery and characterization of RGLS4326, a firstin-class, short oligonucleotide inhibitor of microRNA-17 (miR-17), as a potential treatment for ADPKD. RGLS4326 is discovered by screening a chemically diverse and rationally designed library of anti-miR-17 oligonucleotides for optimal pharmaceutical properties. RGLS4326 preferentially distributes to kidney and collecting duct-derived cysts, displaces miR-17 from translationally active polysomes, and de-represses multiple miR-17 mRNA targets including Pkd1 and Pkd2. Importantly, RGLS4326 demonstrates a favorable preclinical safety profile and attenuates cyst growth in human in vitro ADPKD models and multiple PKD mouse models after subcutaneous administration. The preclinical characteristics of RGLS4326 support its clinical development as a disease-modifying treatment for ADPKD.

\footnotetext{
${ }^{1}$ Regulus Therapeutics Inc., San Diego, CA 92121, USA. ${ }^{2}$ Department of Internal Medicine and Division of Nephrology, University of Texas Southwestern Medical Center, Dallas, TX 75390, USA. Correspondence and requests for materials should be addressed to E.C.L. (email: elee@regulusrx.com) or to V.P. (email: vishald.patel@utsouthwestern.edu)
} 
DPKD is characterized by slowly progressive, bilateral kidney enlargement due to numerous fluid-filled cysts ${ }^{1-3}$. ADPKD is caused by mutations in either PKD1 or PKD2 genes, where disruption of their normal functions leads to excessive proliferation of the renal tubular epithelium causing cyst formation. Over 12 million people worldwide have ADPKD, making it amongst the most commonly known monogenetic disorders. Fifty percent of ADPKD patients eventually develop end-stage renal disease (ESRD) by the age of 60, accounting for $10 \%$ and $5 \%$ of prevalent patients with ESRD in Europe and the United States, respectively. Unfortunately, treatment options for this life-threatening disorder are still limited and inadequate.

miRNAs are evolutionarily conserved, $\sim 20$ nucleotides-long (nt) non-coding RNAs that function as post-transcriptional inhibitors of gene expression. Watson-Crick base pairing between the seed sequence (located primarily in nucleotide $(\mathrm{N})$ position N2-to-N8 at the $5^{\prime}$ end) of the miRNA and the complementary sequences (found mainly in the $3^{\prime}$ untranslated region ( $\left.3^{\prime} \mathrm{UTR}\right)$ ) of mRNAs results in translational repression and eventual degradation of the targeted mRNA transcripts ${ }^{4-6}$. Aberrant activation of miRNAs has been shown to promote the progression of multiple human diseases; therefore, miRNA inhibition has emerged as an attractive therapeutic strategy ${ }^{7-10}$. Anti-microRNA (anti-miRs) are single-stranded, chemically modified oligonucleotides designed to sterically inhibit miRNAs and de-repress downstream target mRNAs and encoded proteins. Indeed, long anti-miRs of 18-21 nt with full complementarity to specific pathogenic miRNAs have been shown to attenuate disease progression in both preclinical and clinical settings ${ }^{11-16}$.

We have recently shown that the miR-17 miRNAs family is upregulated in both human and murine forms of ADPKD, and their deletion or inhibition attenuates cyst growth in mouse PKD models ${ }^{17-19}$. The miR-17 family is derived from three polycistronic clusters: $\mathrm{miR}-17 \sim 92, \mathrm{miR}-106 \mathrm{a} \sim 363$, and $\mathrm{miR}-$ $106 \mathrm{~b} 25$ clusters $^{20}$. Among the three, miR-17 92 is essential for embryonic development and is well-known for its role as an oncogene ${ }^{21,22}$. Germline knockout of miR-17 92 causes perinatal lethality with bone, heart, lung, and $\mathrm{B}$ cell maturation defects, while deletion of the miR-106a 363 or miR-106b 25 clusters does not produce any obvious abnormality ${ }^{23}$. Within the kidney, miR-17 92 deletion in mouse nephron progenitors impairs nephrogenesis ${ }^{24}$. However, the expression of these clusters declines with maturation, and accordingly, inducible deletion of miR-17 92 in adult mice does not impact their lifespan or general well-being, other than reduction in mature hematopoietic lineages ${ }^{25}$. Kidney-specific deletion of the miR17 92 cluster also does not produce any appreciable defects in kidney morphology and histology ${ }^{18}$. Therefore, preferential targeting of the miR-17 family in the post-natal kidney is an attractive therapeutic approach to treat ADPKD.

Based on our previous experience with tool anti-miR-17 oligonucletides ${ }^{17,26,27}$ and known properties of chemically modified oligonucleotides ${ }^{28-30}$, we designed and screened for anti-miR-17 oligonucleotides with favorable pharmaceutical properties that could be used for human clinical testing. Here, we describe the discovery and preclinical evaluation of RGLS4326, a single-stranded, chemically modified, short oligonucleotide of 9-nt with full complementarity to the miR-17 seed sequence. RGLS4326 is designed to preferentially target the kidney and inhibit the pathologic functions of the miR-17 family of miRNAs in ADPKD. Importantly, we report that RGLS4326 reproducibly attenuates cyst growth in human ADPKD models in vitro and multiple PKD mouse models in vivo. Our studies support the clinical development of RGLS4326 for the treatment of ADPKD.

\section{Results}

Discovery of anti-miR-17 oligonucleotide RGLS4326. We discovered RGLS4326 by screening a chemically diverse library of anti-miR-17 oligonucleotides for optimal pharmaceutical properties including potency, stability, safety, pharmacokineticpharmacodynamic profile, and ability to preferentially distribute to kidney and confer therapeutic efficacy following systemic administration (Fig. 1a). The library contained $>190$ oligonucleotides of different lengths, base sequences (complementary to varying positions against mature miR-17), and chemical modifications that were methodically varied to optimize for favorable pharmaceutical profiles. We first screened this library using a miR-17 luciferase sensor assay, where a luciferase reporter vector that contained two fully complementary miR-17 binding sequences in the $3^{\prime} \mathrm{UTR}$ of the luciferase gene was used. Briefly, HeLa cells were co-transfected with the luciferase reporter vector and an exogenous miR-17-expression vector that acted to repress the luciferase signal. Next, HeLa cells were individually treated with anti-miR-17 oligonucleotides from our library. Their ability to sequester miR-17 and de-repress the luciferase signal is plotted in ascending order of potency in Fig. 1b. A subset of these antimiR-17 oligonucleotides with a wide range of chemical designs and in vitro potency against miR-17 were further characterized in normal mice (Fig. 1c) followed by mice with PKD (Fig. 1d). We assessed their ability to engage, inhibit, and displace miR-17 from the translationally active high molecular weight (HMW) polysomes $^{26}$ in the kidney following subcutaneous (SC) administration. We also evaluated their propensity for preferential distribution to kidney over liver while remaining metabolically stable (Fig. 1e, f).

Screening of this library led to the discovery of RGLS4326, a 9nt phosphorothioate backbone-modified oligonucleotide composed of (S)-constrained ethyl, 2'-O-methyl, and 2'-deoxy-2'fluoro nucleoside sugar modifications with a base sequence complementary to the N1-to-N9 position of the mature miR-17 (Fig. 1g). RGLS4326 preferentially distributed to both normal and polycystic kidney, and potently displaced miR-17 from translationally active polysomes in vivo. RGLS4326 was metabolically stable in mouse, monkey, and human tissue lysates and in mouse kidney and liver tissues following SC administration (Supplementary Table 1) and was selected for further evaluation.

RGLS4326 inhibits miR-17 and derepresses direct mRNA targets. We first validated our initial screening results and performed dose-response analysis. RGLS4326 inhibited miR-17 function and de-repressed miR-17 luciferase sensor activity in HeLa cells with an $\mathrm{EC}_{50}$ value of $28.3 \pm 4.0 \mathrm{nM}$ (Fig. 2a). As anticipated from its sequence complementarity to the miR-17 family seed region, RGLS4326 also de-repressed luciferase sensor activity of other miR-17 family members, including miR-20b, miR-93, and miR-106a (Supplementary Fig. 1A-D), but not unrelated miRNAs such as miR-33b (Supplementary Fig. 1E).

In addition to the commonly used luciferase sensor assays, functional inhibition of miRNA is often assessed by measuring de-repression of individual downstream target genes. However, changes in individual miRNA target genes are typically small, variable, and largely cell-type dependent. To circumvent this issue, we developed a mouse miR-17 PharmacodynamicSignature (miR-17 PD-Sig), which consists of the expression of 18 unique miR-17 target genes normalized by six reference housekeeping genes, to provide an unbiased and comprehensive assessment of miR-17 activity. Briefly, four different mouse cell lines (of both kidney and non-kidney origins) were transfected with mock, a chemically diverse set of tool anti-miR-17 oligos, or a tool miR-17-mimic; and whole-transcriptome 
a

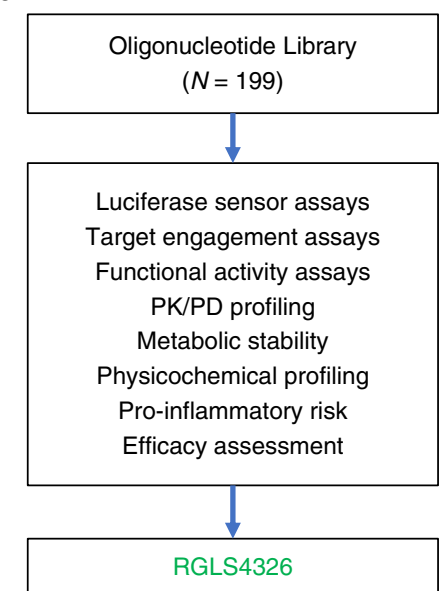

b

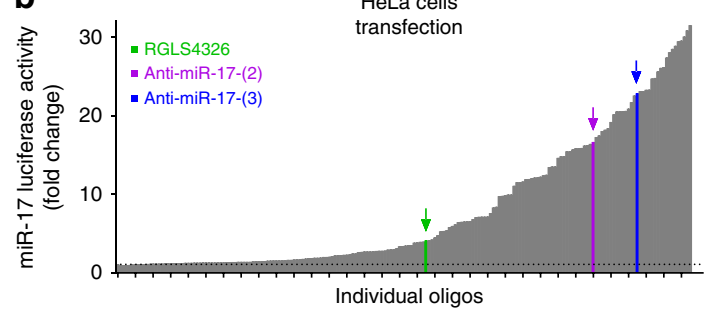

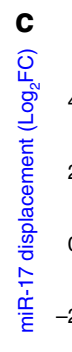

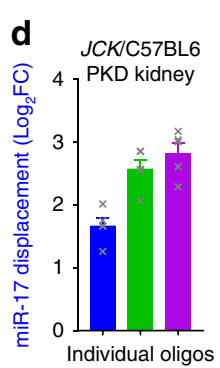

g

mature miR-106b-5p: 5'-UAAAGUGC UGACAGUGCAGAU $-3^{\prime}$ mature miR-106a-5p: 5'-AAAAGUGCUUACAGUGCAGGUAG-3' mature miR-93-5p: 5'-CAAAGUGC UCUUCGUGCAGGUAG-3' mature miR-20b-5p: 5'-CAAAGUGCUCAUAGUGCAGGUAG-3' mature miR-20a-5p: 5'-UAAAGUGCUUAUAGUGCAGGUAG-3' mature miR-17-5p: 5'-CAAAGUGCUUACAGUGCAGGUAG-3' $\|||||||||$

RGLS4326: 3'-GUUUCACGA-5

Red: (S)-constrained ethyl; Blue: 2'-O-methyl; Green: 2'-deoxy-2'-fluoro

Fig. 1 Discovery of RGLS4326, a chemically modified oligonucleotide inhibitor of miR-17. a Screening cascade used for the discovery of RGLS4326. b Over 190 anti-miR-17 oligonucleotides of diverse chemical designs were screened at $10 \mu \mathrm{M}$ in a miR-17 HeLa cell luciferase assay and plotted in ascending order of potency ( $n=1$ /oligonucleotides). Selected oligonucleotides including RGLS4326 (green) are highlighted for illustration purposes. $\mathbf{c}$ A subset of oligonucleotides was tested in WT/C57BL6 mice for their ability to engage and displace miR-17 in the kidney 7 days after a single $30 \mathrm{mg} \mathrm{kg}-1$ SC dose ( $n=$ 4). d A smaller set of oligonucleotides were further tested in the JCK/C57BL6 PKD model for miR-17 target engagement $(n=5)$. e-f Preferential distribution to kidney over liver 7 days after a single $30 \mathrm{mg} \mathrm{kg}^{-1}$ SC dose of selected oligonucleotides in WT/C57BL6 $(n=4)$ and JCK/C57BL6 mice $(n=$ 5) are shown. $\mathbf{g}$ Chemical modifications, base sequence, and corresponding complementarity to the miR-17 family of mature miRNAs for RGLS4326 is illustrated. Error bars represent standard error of means. Source data for Fig. $1 \mathbf{b}-\mathbf{f}$ is provided in Source data files

RNA-sequencing was performed. Direct miR-17 target genes (containing $3^{\prime}$ UTR sequences complementary to miR-17 family seed) whose expression increased after anti-miR-17 treatment but decreased after miR-17-mimic treatment were ranked, filtered for non-redundancy, and selected as candidate genes (Supplementary Fig. 2A). These candidate genes were further validated by $\mathrm{qPCR}$ in one additional non-kidney cell line before the final top 18 ranked genes were chosen (Supplementary Fig. 2B-C). The resultant mouse miR-17 PD-Sig score is the calculated average of the 18 genes' individual $\log _{2}$ fold changes (normalized by six housekeeping genes) compared to mock. Indeed, as exemplified in Supplementary Fig. 2D, our miR-17 PD-Sig can be used to comprehensively and unbiasedly assess miR-17 activity within a dynamic range that allows for rankordering of both anti-miR-17 and miR-17 mimic of interest.

We next utilized this mouse miR-17 PD-Sig to evaluate the activity of RGLS4326. RGLS4326 treatment inhibited miR-17 function in kidney collecting duct cells in culture as measured by miR-17 PD-Sig, with an $\mathrm{EC}_{50}$ value of $77.2 \pm 20.2 \mathrm{nM}$ (Fig. 2b). The direct miR-17 target genes $P k d 1$ and $P k d 2$, which are central to ADPKD pathogenesis ${ }^{2}$ were also de-repressed (Fig. 2c, d). De-repression of miR-17 PD-Sig after RGLS4326 treatment was not restricted to collecting duct cells, as the same was also observed in six different mouse kidney cell lines of proximal tubule, distal tubule and collecting duct origins derived from normal and PKD mouse kidneys (Fig. 2e), and a mouse kidney slice culture ex vivo (Fig. 2f).

As previously demonstrated, functional inhibition of miR-17 can also be assessed by measuring the amount of miR-17 displaced from the translationally active HMW polysomes by miRNA polysome shift assay ${ }^{26}$ following anti-miR-17 treatment. We therefore evaluated miR-17 PD-Sig as readout of RGLS4326 activity in a head-to-head comparison with the miRNA polysome shift assay. As shown in Supplementary Fig. 3, we observed a positive and dose-dependent correlation between the displacement of miR-17 and de-repression of miR-17 PD-sig after RGLS4326 treatment. In contrast, treatment with control oligonucleotides had no effect. Taken together, our comprehensive screening cascade has enabled the discovery of RGLS4326 that engages and displaces miR-17 from translationally actively HMW polysomes thereby de-repressesing direct miR-17 target mRNAs.

RGLS4326 suppresses the growth of primary human ADPKD cysts. We next examined the therapeutic efficacy of RGLS4326 in vitro. To demonstrate human translational potential, we utilized primary cyst cultures derived from human ADPKD donors. We first demonstrated that RGLS4326 treatment globally derepressed mRNAs of predicted miR-17 target genes (Fig. 3a), as 

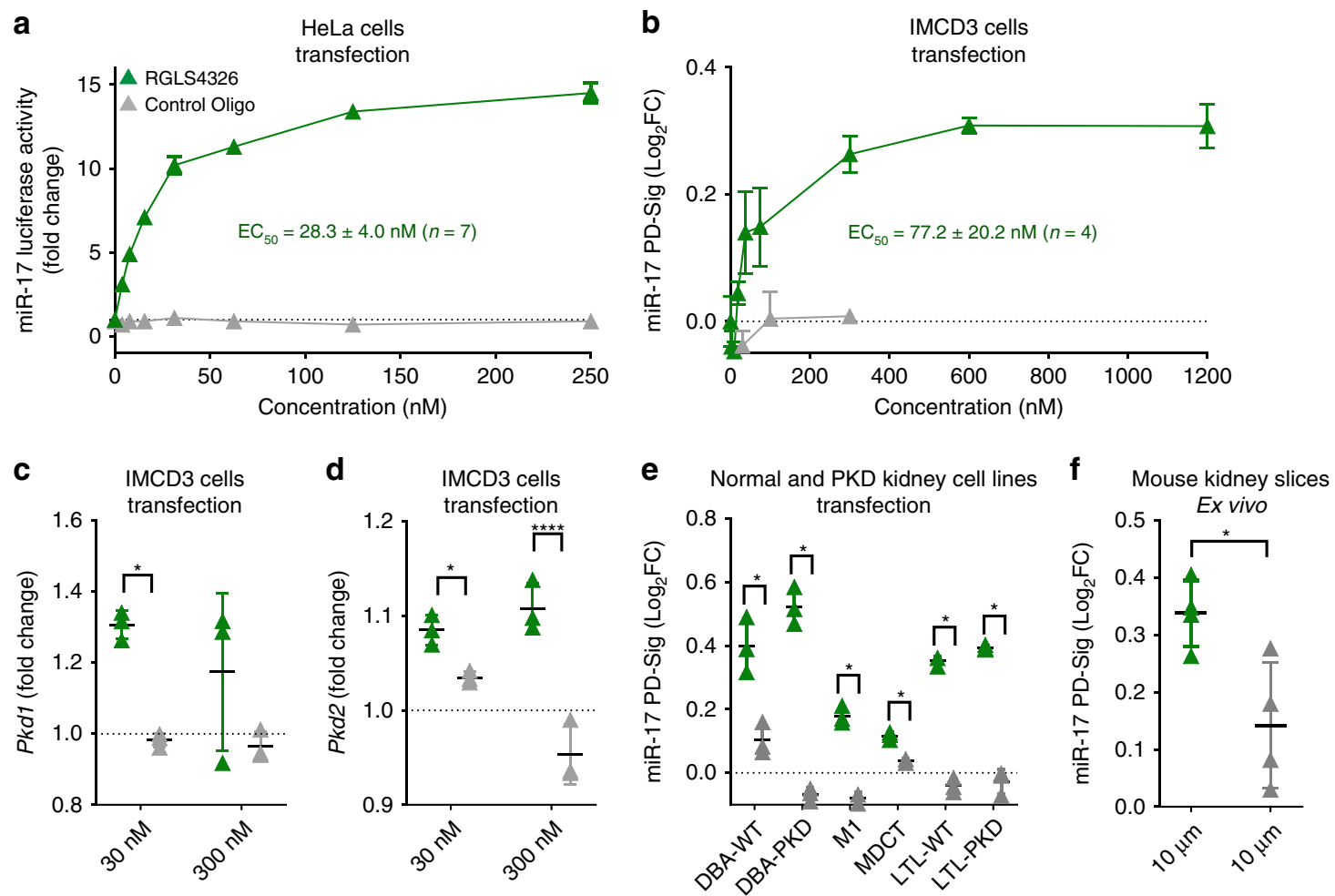

Fig. 2 RGLS4326 inhibits miR-17 and de-represses direct miR-17 targets. a RGLS4326 (green triangle) dose-responsively inhibits miR-17 in HeLa cell luciferase assay $24 \mathrm{~h}$ after transfection, with an $\mathrm{EC}_{50}$ value of $28.3 \pm 4.0 \mathrm{nM}$ (mean \pm standard deviation, $n=7$ independent experiments). $\mathbf{b}$ RGLS4326 dose-responsively de-represses multiple miR-17 target genes (as measured by miR-17 PD-Sig) in mouse IMCD3 cells $24 \mathrm{~h}$ after transfection, with an EC 50 value of $77.2 \pm 20.2 \mathrm{nM}$ ( $n=4$ independent experiments). c-d RGLS4326 treatment results in de-repression of the direct miR-17 target genes, Pkd1 and Pkd2 in IMCD3 cells $(n=3)$. e RGLS4326, but not control oligo, functionally inhibited miR-17 and de-repressed miR-17 PD-Sig in six cell lines derived from normal (DBA-WT, M1, MDCT, LTL-WT) or PKD (DBA-PKD and LTL-PKD) mouse kidneys after $24 \mathrm{~h}$ treatment by transfection at $30 \mathrm{nM}(n=3)$ and in $\mathbf{f}$ normal mouse kidney tissue slice culture after $72 \mathrm{~h}$ ex vivo incubation at $10 \mu \mathrm{M}(n=4)$. Control oligo (grey triangle) containing the same chemicalmodification, length, and design as RGLS4326, but different base pair sequence, was used as a negative control. Error bars represent standard deviations. ${ }^{\star} p<0.05,{ }^{\star \star \star \star} p<0.0001$. One-way ANOVA, Dunnette's multiple comparison test. Source data for Fig. 2a-f is provided in Source data files

well as the previously defined human miR-17 PD-Sig 27 (Fig. 3b) in human primary ADPKD cysts. Moreover, expression of the direct miR-17 target encoded proteins polycystin-1 (PC1) and polycystin-2 (PC2) increased by $\sim 2$-fold and 4-fold, respectively, after RGLS4326 treatment (Fig. 3c). Next, we measured its effect on 3D-cyst growth and proliferation. Culturing of RGLS4326treated primary cysts in 3D Matrigel showed a significant reduction of in vitro cyst growth and proliferation in a concentration-dependent manner (Fig. 3d-f). While the expected low magnitude of non-specific and idiosyncratic changes in PDSig and cyst growth were observed following control oligonucleotide treatment, our data from two individual human ADPKD donor cyst cultures demonstrate a positive correlation between miR-17 inhibition and cyst growth reduction across all treatments (Fig. 3g, h). Moreover, this effect is specific to ADPKD cells, as we observed no cytotoxicity in RGLS4326-treated non-ADPKD kidney cells where miR-17 inhibition was evident (Supplementary Fig. 3).

RGLS4326 preferentially distributes to kidney tubules and cysts. We next evaluated the in vivo pharmacokinetic and biodistribution profile of RGLS4326 in wild-type mice following a single $30 \mathrm{mg} \mathrm{kg}^{-1} \mathrm{SC}$ injection. RGLS4326 is rapidly absorbed into plasma, showing $\mathrm{T}_{\max }$ of $\leq 1 \mathrm{~h}, \mathrm{C}_{\max }$ of $8.5 \mu \mathrm{g} \mathrm{mL}^{-1}$, and halflife of $<4 \mathrm{~h}$ (Fig. $4 \mathrm{a}$ ). The rapid plasma clearance of RGLS4326 reflects the extensive distribution to tissues suchs as kidney and liver, that is commonly observed for chemically modified oligonucleotides ${ }^{29}$. Uniquely, RGLS4326 showed preferential kidney distribution, with kidney-to-liver $(\mathrm{K} / \mathrm{L})$ ratio of approximately 13 and 8 by $C_{\max }$ and $\mathrm{AUC}_{\text {last }}$, respectively (Fig. $4 \mathrm{~b}$ ). Data from a quantitative whole-body autoradiography (QWBA) study further demonstrated that RGLS4326 primarily distributes to kidney over any other organs after a single SC dose (Fig. 4c), with the majority of the administered dose eliminated in the urine. Within the kidney, chemically modified oligonucleotides are known to localize principally to proximal tubules by basolateral uptake and tubular reabsorption, whereas localization in glomeruli is mini$\mathrm{mal}^{11,30}$. Indeed, following subcutaneous administration in wildtype mice, RGLS4326 was present mostly in proximal tubules (Fig. 4d), with lower amounts detected in cortical collecting ducts (Fig. 4e), and no glomerular uptake was noted. Importantly, we observed extensive RGLS4326 uptake in both proximal tubules and collecting duct cysts in cystic kidneys of the aggressive Pkhd1/cre; $P k d 2^{\mathrm{F} / \mathrm{F}}(P k d 2-\mathrm{KO})$ mouse model of ADPKD following SC administration, likely linked to the severely disrupted kidney architecture in this mouse model (Fig. 4e). Additional representative immunofluorescence images of stained kidney sections are shown in Supplementary Figs. 4 and 5.

We next studied the ability of RGLS4326 to inhibit endogenous miR-17 in kidneys. Consistent with our observations of rapid kidney distribution, RGLS4326 started displacing miR-17 from HWM polysomes within $24 \mathrm{~h}$ after a single SC administration, with peak displacement occurring on day 7 and continuing for at least 14 days (Fig. 4f). To further examine the in vivo pharmacodynamic profile, various doses of RGLS4326 were subcutaneously administered to normal and PKD mice (JCK/ 

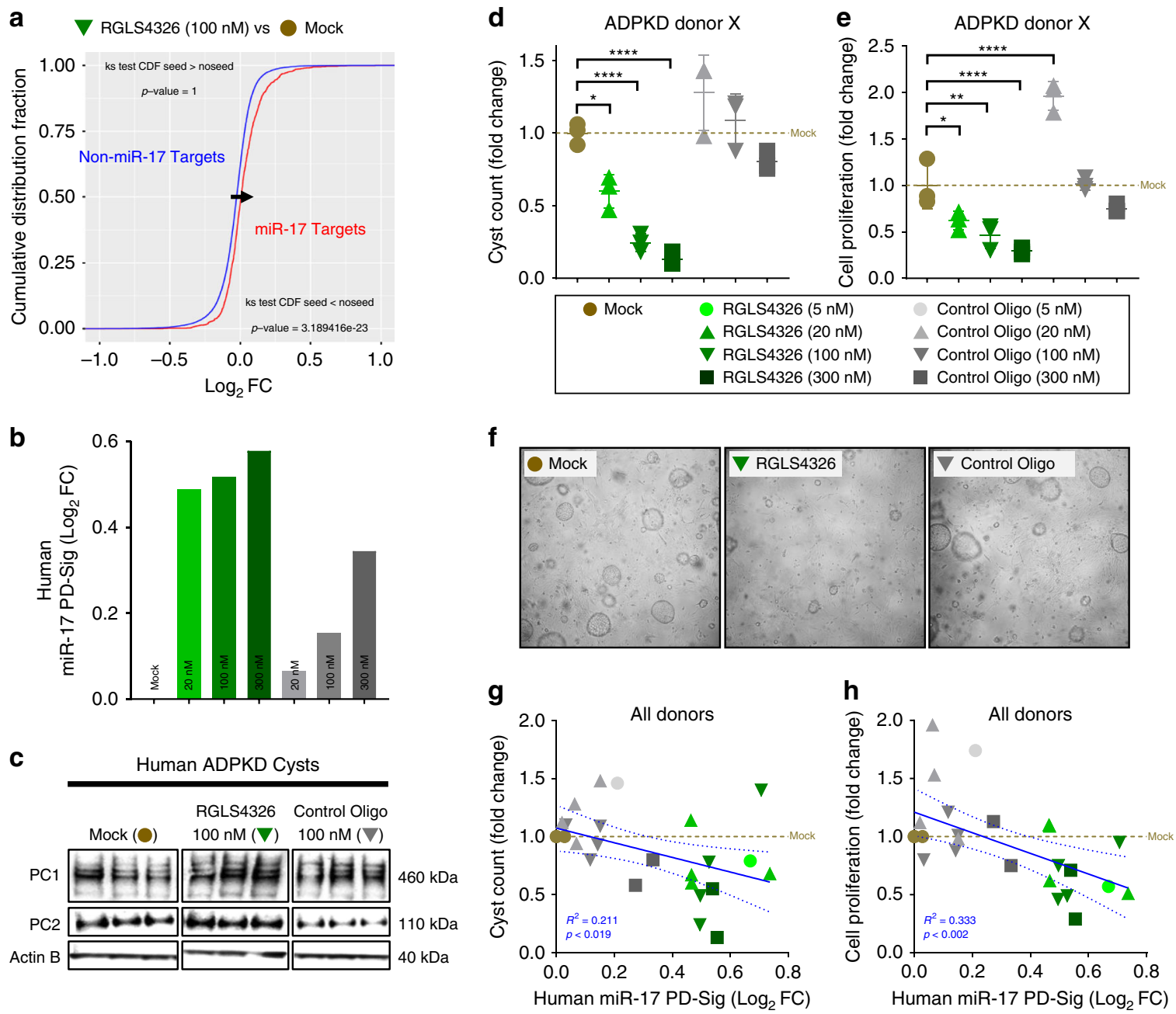

Fig. 3 RGLS4326 suppresses the growth of primary human ADPKD cysts in vitro. a Primary cyst cultures derived from human ADPKD donors were transfected with RGLS4326 at $100 \mathrm{nM}$ for $24 \mathrm{~h}$ and harvested for RNA-seq analysis. Kolmogorov-Smironov test statistics comparing the cumulative distribution of global mRNA changes between RGLS4326-treated (inverted green triangle) vs. mock-treated (brown circle) samples indicated significant de-repression of predicted miR-17 target genes (as defined by TargetScanHuman v7.1) after RGLS4326 treatment. b For each subsequent experiment, functional inhibition of miR-17 was assessed by measuring de-repression of human miR-17 PD-Sig 27 in representative cyst samples ( $n=1 /$ treatment/dose) after $24 \mathrm{~h}$ transfection with RGLS4326 (shades of green) or control oligo (shades of grey) and prior to further culturing in 3D Matrigel for an additional 8 days. c Western blot analysis demonstrating increased expression of polycystin-1 (PC1) and polycystin-2 (PC2) $72 \mathrm{~h}$ following RGLS4326 treatment ( $n=$ 3). d-e Quantification and (f) representative images of 3D cyst formation showing a reduction in cyst count and proliferation 9 days following initial RGLS4326 treatment $(n=3)$. $\mathbf{g}-\mathbf{h}$ Inhibition of miR-17 as measured by miR-17 PD-Sig correlates with anti-cyst and anti-proliferation activity $(n=4$ independent experiments among 2 donors). $R^{2}, p$-values and $95 \%$ confidence interval limits (dotted lines) from corresponding linear regression models were shown. Error bars represent standard deviations. ${ }^{\star} p<0.05,{ }^{\star \star} p<0.01,{ }^{\star \star \star \star} p<0.0001$. One-way ANOVA, Dunnette's multiple comparison test. Source data for Fig. $3 \mathbf{b}-\mathbf{e}$, and $\mathbf{g}-\mathbf{h}$ is provided in Source data files

C57BL6 and Pcy/CD1). RGLS4326 treatment dose-responsively displaced kidney miR-17 from HMW polysomes in both normal and PKD mice (Fig. 4g-i). In contrast, treatment with PBS or control oligonucleotides had no effect. Our analysis showed that 7 -days after a single $30 \mathrm{mg} \mathrm{kg}^{-1}$ SC injection, RGLS4326 potently displaced kidney miR-17 with observed miPSA scores of 3.16, 2.58 , and 2.56, representing calculated percent inhibition of 89 , 83 , and $83 \%$ in normal, JCK/C57BL6 and Pcy/CD1 mice, respectively.

RGLS4326 confers efficacy in multiple PKD mouse models. The rapidly progressing $P k d 2$-KO mouse model of ADPKD is responsive to miR-17 inhibition, where genetic knockdown of miR-17 92 attenuates disease progression ${ }^{17}$. Therefore, we sought to determine whether RGLS4326 treatment produces similar beneficial effects in a randomized, blinded and statistically powered efficacy study in $P k d 2-\mathrm{KO}$ mice. $P k d 2-\mathrm{KO}$ mice were dosed SC with PBS, $20 \mathrm{mg} \mathrm{kg}^{-1}$ of RGLS4326 or control oligo at post-natal day (P)10, P11, P12, and P19; and kidneys were harvested on P13, P16, P19, and P28. Compared to non-cystic control kidneys, polycystic kidneys of PBS-treated Pkd2-KO mice exhibit an age-dependent progressive decline in miR-17 PD-Sig, indicative of increasing miR-17 activity with disease progression (Fig. 5a). Administration of RGLS4326 reversed this decline in miR-17 PD-Sig, indicating a sustained functional inhibition of miR-17. Like previous in vitro studies, in vivo administration of RGLS4326 also upregulated the expression of the direct miR-17 target genes $P k d 1$ and $P k d 2$ (Fig. 5b, c). Most importantly, RGLS4326 treatment led to reduction in kidney-to-body-weight ratio $(\mathrm{KW} / \mathrm{BW})$ and decrease in cyst epithelial cell proliferation (Fig. 5d-f). In contrast, no effect was observed with the control oligonucleotide. 
a

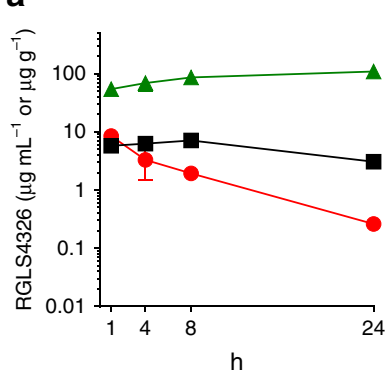

b

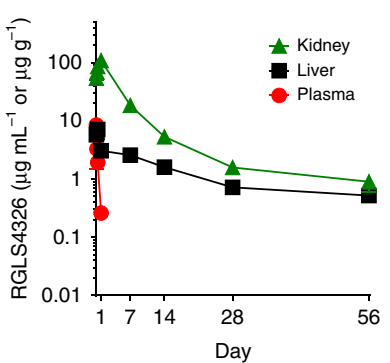

C

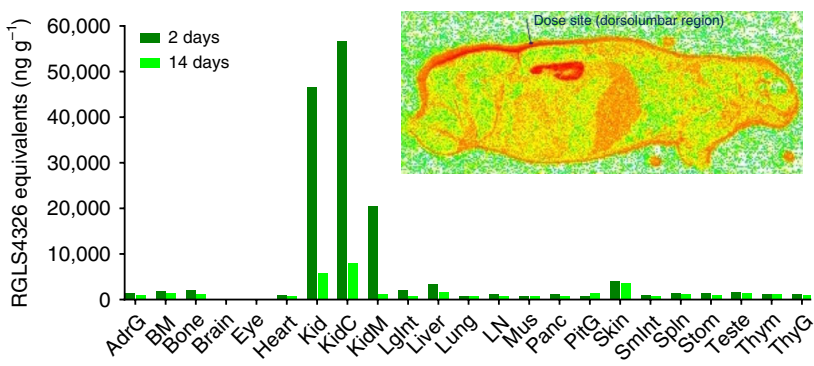

d

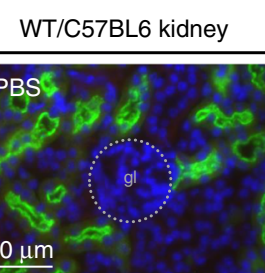

Pkd2-KO kidney

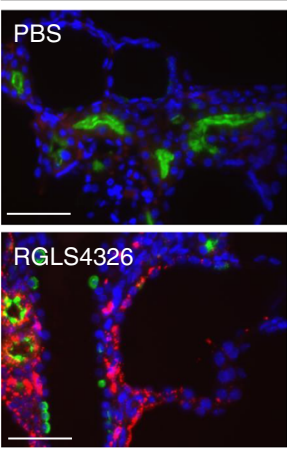

e

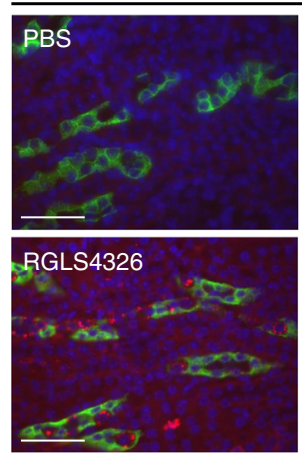

WT/C57BL6 Kidney
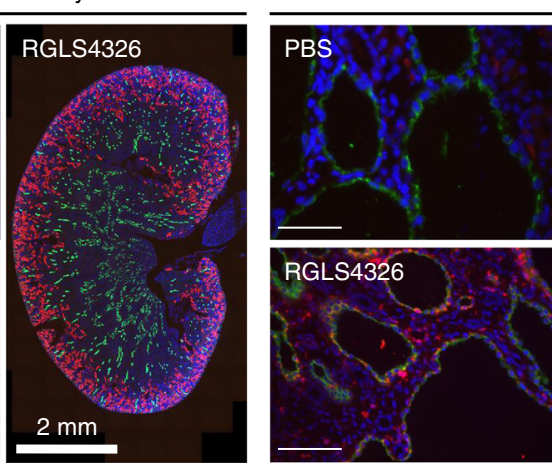

Pkd2-KO kidney

DBA; $\square$ Anti-PS Ab; $\square$ DAPI

LTA; Anti-PS Ab; DAPI
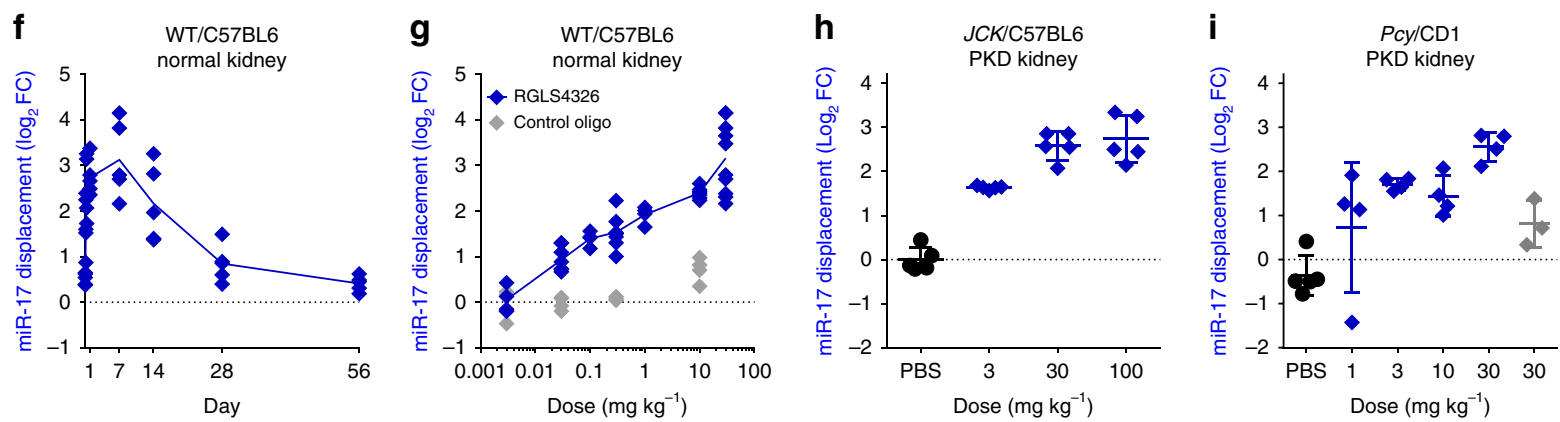

Fig. 4 Pharmacokinetic, tissue distribution and pharmacodynamic profile of RGLS4326. a-b Kidney (green triangle), liver (black square) and plasma (red circle) exposures-vs.-time profiles of RGLS4326 following a single $30 \mathrm{mg} \mathrm{kg}^{-1} \mathrm{SC}$ dose in WT/C57BL6 mice $(n=5 /$ group). c Tissue distribution profile of RGLS4326 showing preferential kidney distribution based on quantitative whole-body autoradiography of [35S]-RGLS4326-derived radioactivity in male WT/CD1 mice 2 days (dark green) and 14 days (light green) after a single SC dose of RGLS4326 at $30 \mathrm{mg} \mathrm{kg}^{-1}$ and target radioactivity of $100 \mu \mathrm{Ci} \mathrm{kg}{ }^{-1}$ $(n=1$ /timepoint). Representative whole-body autoradioluminogram showing tissue distribution of radioactivity at Day 2 is shown. Red indicates intensity of radioactivity detected. d-e WT/C57BL6 $(n=3)$ or Pkd2-KO mice $(n=3)$ were dosed SC with PBS or $20 \mathrm{mg} \mathrm{kg}^{-1}$ of RGLS4326 on postnatal day (P)21, P22 and P23, and kidneys were harvested on P26. Kidney sections were co-stained with LTA (proximal tubules marker) or DBA (collecting ducts marker), anti-PS antibody (antibody labels RGLS4326) and DAPI. Representative merged immunofluorescence images of stained kidney sections demonstrating delivery of RGLS4326 to (d) proximal tubules and (e) collect duct cyst cells are shown. No glomerulus localization of RGLS4326 was observed in all mice tested. $\mathbf{f}$ Kidney target engagement-vs.-time profile of RGLS4326 showed kidney target engagement (blue diamond) peaked at 7 days, and continued through to at least 14 days, after a single $30 \mathrm{mg} \mathrm{kg}^{-1} \mathrm{SC}$ dose in WT/C57BL6 mice ( $n=5 /$ group). $\mathbf{g}$ Dose-responsive target engagement of miR-17 in kidney tissues 7 days following a single SC dose of RGLS4326 at $0.003(n=4), 0.03(n=8), 0.1(n=4), 0.3(n=8), 3(n=4), 10(n=8)$, and $30 \mathrm{mg} \mathrm{kg}-1$ $(n=10)$ in WT/C57BL6 mice. h-i RGLS4326 treatment dose-responsively engaged miR-17 in polycystic kidneys of two PKD mouse models 7 days after a single SC dose compare to PBS (closed black circle). RGLS4326 was dosed at 3, 30, and $100 \mathrm{mg} \mathrm{kg}^{-1}$ in JCK/C57BL6 mice ( $n=5$ ), and at 1, 3, 10, $30 \mathrm{mg} \mathrm{kg}^{-1}$ in Pcy/CD1 mice $(n=4)$. Control oligo (grey) was used as a negative control. Error bars represent standard deviations. Glomerulus, $g /$ (dotted circle). Scale bars, $50 \mu \mathrm{m}$ (except as specified in e, $2 \mathrm{~mm}$ ). Source data for Fig. $4 \mathbf{a}-\mathbf{c}$ and $\mathbf{f - i}$ is provided in Source data files

RGLS4326 was then evaluated in three independent long-term studies. First, we performed a randomized and statistically powered efficacy study in the slowly progressing Pcy/CD1 mouse model (Fig. 5g). Five-weeks-old Pcy/CD1 mice were treated with RGLS4326 at $25 \mathrm{mg} \mathrm{kg}^{-1}$ for 25-weeks. Reduction of KW/BW, cyst index, urine Ngal protein and kidney Ngal mRNA expression were observed after either weekly (QW) or monthly (Q4W) SC dosing of RGLS4326 (Fig. 5h-k). Treatment with control oligonucleotides had no effect. Initiating therapy in 15-weeksold $P c y / \mathrm{CD} 1$ mice (QW late) did not attenuate cyst growth, exemplifying the importance of early treatment initiation in this model.

In a second long-term study, we examined RGLS4326 in the more aggressive Pcy/DBA mouse model ${ }^{31}$. Six-weeks-old Pcy/ DBA mice were treated with QW doses of RGLS4326 at 25, 5, or $1 \mathrm{mg} \mathrm{kg}^{-1}$ for 9 -weeks. Tolvaptan is currently the only approved 
a

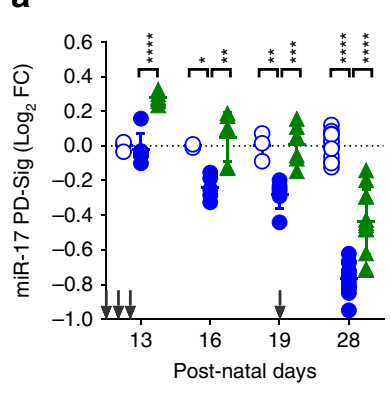

d
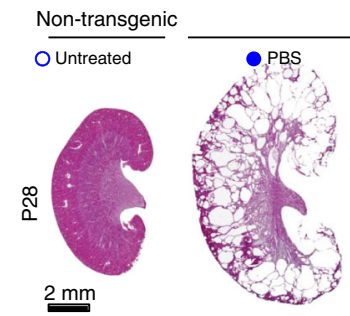

Pkd2-KO
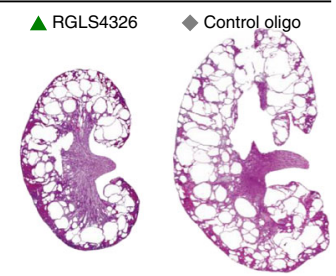

e

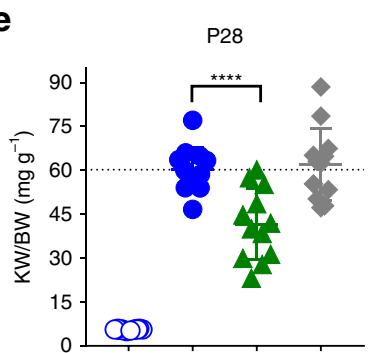

b

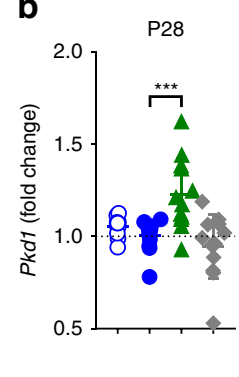

f

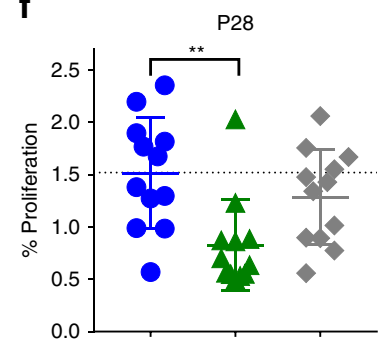

C

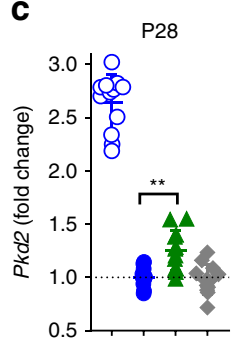

g $W T / C D 1$
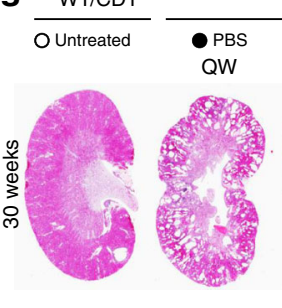

Pcy/CD1

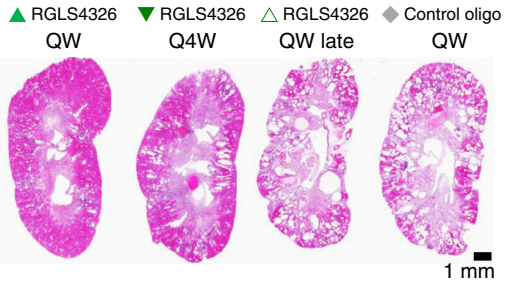

h

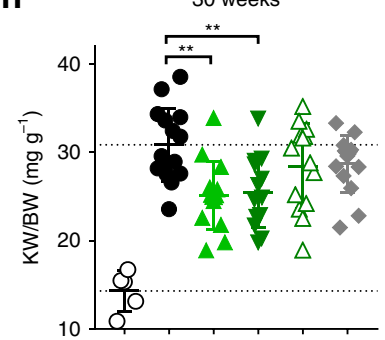

i $\mathbf{j}$

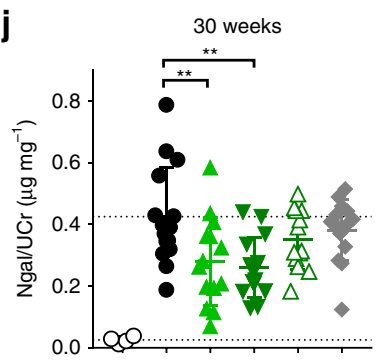

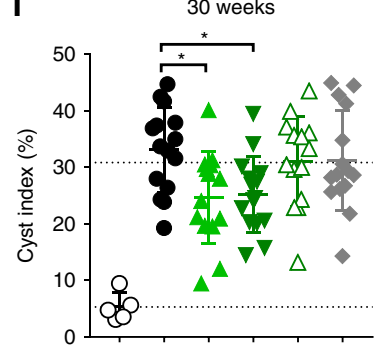

k

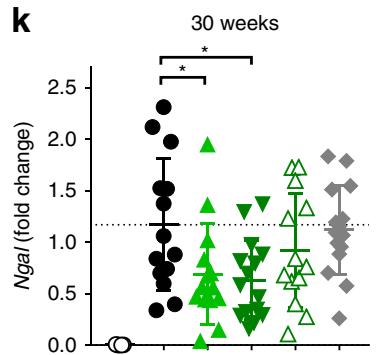

Fig. 5 RGLS4326 confers efficacy in Pkd2-KO and Pcy/CD1 mouse models. a-f Pkd2-KO mice were dosed SC with PBS (closed blue circle) or 20 mg kg ${ }^{-1}$ of RGLS4326 (green triangle) or control oligo (grey diamond) at P10, P11, P12, and P19. Kidneys were harvested on P13, P16, P19 ( $n=6 / g r o u p)$, and P28 ( $n=$ 12 ), and total RNA was extracted. Age-matched untreated non-transgenic (UNT) control mice were also included for analysis (open blue circle; $n=3$ for P13, P16, and P19 and $n=12$ for P28). a Pkd2-KO kidneys show low level of miR-17 PD-sig, indicative of higher baseline miR-17 functional activity compared to UNT. RGLS4326 treatment de-repressed miR-17 PD-Sig. Arrows indicate dosing days. b-c RGLS4326 treatment results in de-expression of Pkd1 and Pkd2. d Representative H\&E staining of kidney sections from each treatment groups at the end of the study on P28. e Kidney-weight-to-body-weight ratio (KW/BW), as well as (f) number of proliferating cyst epithelial cells (as stained by anti-pHH3 antibody) were reduced after RGLS4326 treatment. g-k Five-weeks-old male Pcy/CD1 mice were dosed SC once-weekly (QW) with PBS (closed black circle) or $25 \mathrm{mg} \mathrm{kg}^{-1}$ of RGLS4326 (green triangle) or control oligos (grey diamond), or once-every-4-weeks (Q4W) with $25 \mathrm{mg} \mathrm{kg}^{-1}$ of RGLS4326 (inverted green triangle). An additional group of Pcy/CD1 mice were dosed SC once-weekly with $25 \mathrm{mg} \mathrm{kg}^{-1}$ of RGLS4326 starting at 15-weeks of age (QW late; open green triangle). All Pcy/CD1 mice ( $n=15 /$ group) were euthanized at 30-weeks of age, and kidney and urine samples were harvested. $\mathbf{g}$ Representative H\&E staining of kidney sections from each treatment groups at the end of the study are shown. $\mathbf{h} \mathrm{KW} / \mathrm{BW}$ ratio, (i) cyst index, (j) urine Ngal protein, and (k) kidney Ngal mRNA expression were significantly reduced in Pcy/CD1 mice following RGLS4326 treatment. Corresponding values from wild-type controls $(n=5)$ are shown for reference (open black circle). Error bars represent standard deviations. ${ }^{\star} p<0.05,{ }^{\star \star} p<0.01,{ }^{\star \star \star} p<0.001,{ }^{\star \star \star \star} p<0.0001$. One-way ANOVA, Dunnette's multiple comparison test. Scale bars, $2 \mathrm{~mm}(\mathbf{d})$ and $1 \mathrm{~mm}(\mathbf{g})$. Source data for Fig. $5 \mathbf{a}-\mathbf{c}, \mathbf{e}, \mathbf{f}$, and $\mathbf{h}-\mathbf{k}$ is provided in Source data files

treatment for ADPKD. Therefore, we also performed a head-tohead comparison between RGLS4326 and tolvaptan in the Pcy/ DBA model. RGLS4326 demonstrated a dose-dependent reduction in KW/BW and cyst index following 9 consecutive weeks of QW SC injections (Fig. 6a-c). However, monthly RGLS4326 treatment did not confer efficacy in the Pcy/DBA model. Tolvaptan treatment reduced KW/BW but did not improve cyst index in this head-to-head comparison.

Finally, in the third long-term study, we prospectively monitored disease progression in $P c y / \mathrm{DBA}$ mice treated with either PBS or $30 \mathrm{mg} \mathrm{kg}^{-1}$ RGLS4326 SC QW. Disease progression in $\mathrm{ADPKD}$ patients can be tracked by height-adjusted total kidney volume (htTKV) using magnetic resonance imaging $(\mathrm{MRI})^{32,33}$. Hence, we monitored Pcy/DBA mice using the comparable MRI-based body-weight-adjusted TKV (bwTKV) (Fig. 6d). RGLS4326 treatment was initiated in 7-week-old Pcy/ DBA mice in which substantial cysts had already formed. In an 8-week efficacy study, bwTKV increased almost linearly in
PBS-treated Pcy/DBA mice, whereas growth of polycystic kidneys started to plateau after only 4 weeks of RGLS4326 treatment (Fig. 6e). One out of ten Pcy/DBA mice treated with RGLS4326 showed stabilization and two out of ten mice showed a reduction of bwTKV.

RGLS4326 improves dysregulated gene network expression in PKD. We have shown that RGLS4326 displaces miR-17 from the translationally active HMW polysomes, de-represses miR-17 target genes including $P k d 1$ and $P k d 2$ and their encoded proteins PC1 and PC2, and is efficacious in PKD models (Fig. 7a). To gain further insights into RGLS4326 mechanism-of-action (MOA) in the context of PKD treatment, we began by comparing the kidney global mRNA expression profiles in the $P k d 2-\mathrm{KO}$ and $P c y / \mathrm{CD} 1$ models following RGLS4326 treatment vs. their respective PBStreated controls. We found that RGLS4326 treatment had a significant transcriptome-wide impact and globally de-repressed 
a

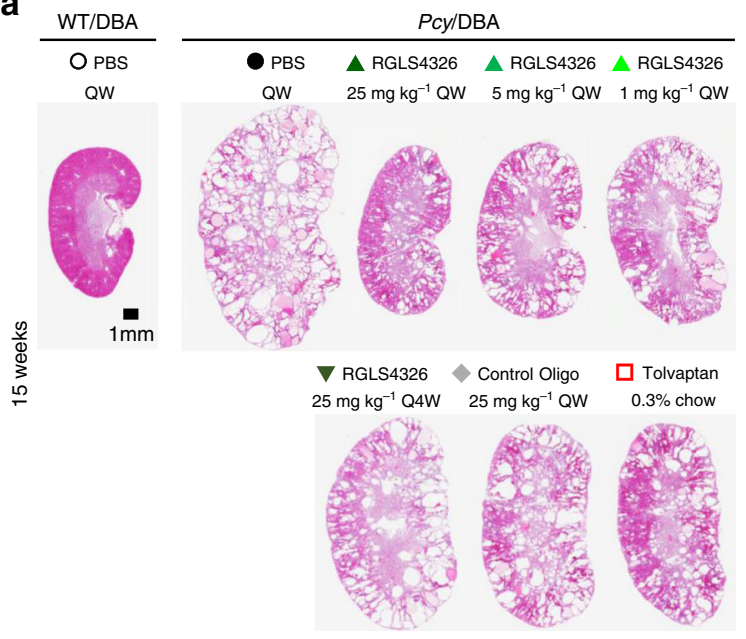

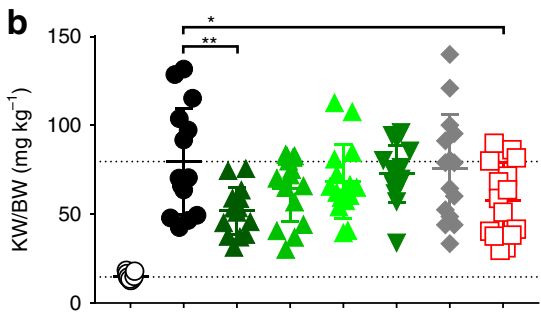

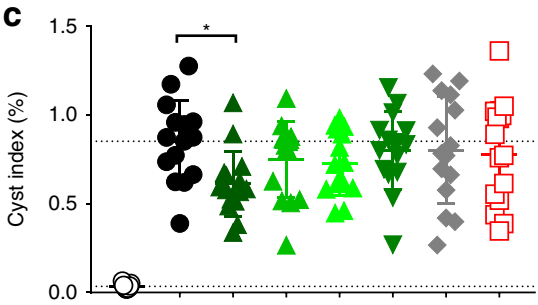

d

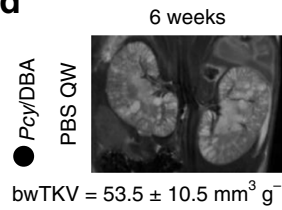

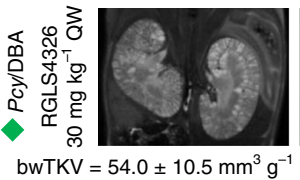

8 weeks
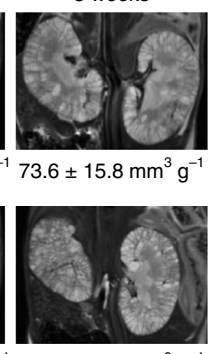

11 weeks

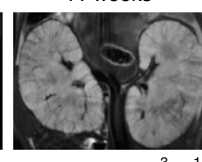

$89.8+14.3 \mathrm{~mm}^{3} \mathrm{~g}^{-1}$

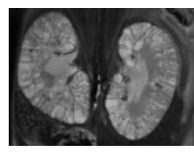

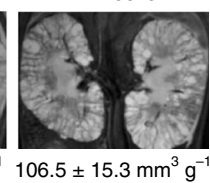

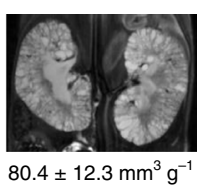

e

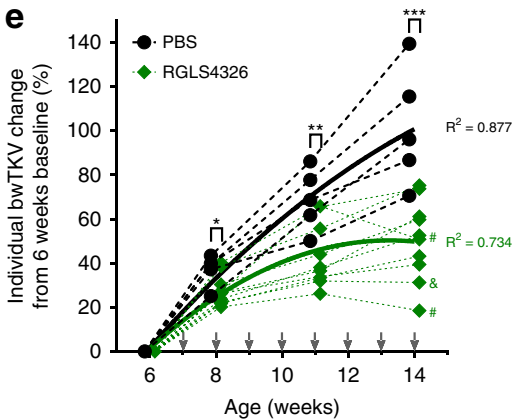

Fig. 6 RGLS4326 confers efficacy in Pcy/DBA mouse model. a-c Six-weeks-old Pcy/DBA mice were dosed SC QW with PBS (closed black circle), or 25 mg $\mathrm{kg}^{-1}$ (dark green triangle), $5 \mathrm{mg} \mathrm{kg}^{-1}$ (green triangle) or $1 \mathrm{mg} \mathrm{kg}^{-1}$ of RGLS4326 (light green triangle), or Q4W with $25 \mathrm{mg} \mathrm{kg}{ }^{-1}$ of RGLS4326 (inverted green triangle), or QW with $25 \mathrm{mg} \mathrm{kg}^{-1}$ of control oligos (grey diamond). Another group of 6-weeks-old Pcy/DBA mice was treated with $0.3 \%$ tolvaptan via diet ad libitum (open red square). All Pcy/DBA mice ( $n=15 /$ group) were euthanized at 15-weeks of age, and kidneys were harvested. a Representative H\&E staining of kidney sections from each treatment groups are shown. $\mathbf{b} \mathrm{KW} / \mathrm{BW}$ ratio and (c) cyst index were significantly reduced in Pcy/DBA mice following weekly RGLS4326 treatment. Tolvaptan conferred efficacy based on KW/BW ratio, but not cyst index. Corresponding values from PBS-treated WT/DBA mice are shown for reference (open black circle, $n=5$ ). d Baseline body-weight-adjusted total kidney volume (bwTKV) were obtained from 6weeks-old male Pcy/DBA mice by T2-weighted MRI and used for treatment group randomization. Assigned mice were dosed SC QW with PBS ( $n=5$; closed black circle), or $30 \mathrm{mg} \mathrm{kg}{ }^{-1}$ RGLS4326 ( $n=10$; green diamond). Representative MRI images and mean bwTKV \pm standard deviations from 6-, 8-, 11-, and 14-week-old mice from each treatment groups are shown. e Percentage change of individual bwTKV changes from 6 weeks baseline values for each timepoints are shown. The bwTKV-vs.-time profiles for each treatment groups were fitted with second-order polynomial regression for illustration purposes. $\mathrm{R}^{2}$ values are shown. Arrows indicate dosing days. Pcy/DBA mice with stabilized and reduced bwTKV from last measurements at the end of the study are indicated by $\&$ and \#, respectively. Error bars represent standard deviations. ${ }^{\star} p<0.05,{ }^{\star \star} p<0.01,{ }^{\star \star \star} p<0.001$. One-way ANOVA, Dunnette's multiple comparison test. Scale bar, $1 \mathrm{~mm}$. Source data for Fig. $6 \mathbf{b}, \mathbf{c}$ and $\mathbf{e}$ is provided in Source data files

mRNAs of predicted miR-17 target genes in cystic kidneys of both PKD models (Fig. 7b, c). In parallel, we also compared kidney expression profiles of the two PKD mouse vs. their respective non-diseased strain-matched controls. As expected, numerous genes were aberrantly expressed in cystic kidneys of these mice compared to their non-diseased counterparts (Fig. 7d, e; $x$-axis). Comparative differential expression analysis showed a clear trend in global transcriptomic profiles where the expressions of dysregulated genes in the $P k d 2-\mathrm{KO}$ and $P c y / \mathrm{CD} 1$ cystic kidneys were improved after RGLS4326 treatment (rho $=-0.559$ and -0.812 , respectively) (Fig. $7 \mathrm{~d}, \mathrm{e} ; y$-axis). Specifically, RGLS4326 treatment improved the expression of a total of 994 genes in $P k d 2-\mathrm{KO}$ kidneys and 658 genes in $P c y / \mathrm{CD} 1$ kidneys $\left(\mathrm{FDR}<0.05\right.$ and $\log _{2} \mathrm{FC}>|0.5|$ ). We next used ingenuity pathway analysis software to identify gene networks and pathways that were improved in the PKD models following RGLS4326 treatment. The top 15 pathways potentially responsible for the gene changes are shown in Fig. 7f. Consistent with our previous observations in miR-17 92 genetic deletion studies and the known oncogenic role of miR-17, RGLS4326 treatment was associated with normalization of metabolism pathways ${ }^{34}$ (e.g., PPAR $\alpha / R X R \alpha)$ and inhibition of pro-proliferative oncogenic pathways $^{21}$ (e.g., Wnt pathway).

Preclinical safety assessment of RGLS4326. Oligonucleotides containing 2 '-deoxy-2'-fluoro nucleosides have the potential to inhibit DNA repair and to induce mitochondrial toxicity ${ }^{35-37}$. We therefore assessed the genotoxic and mitochondrial toxicity potential of RGLS4326, which contains three 2 -deoxy- 2 '-fluoro nucleosides. No genotoxicity was observed in bacterial mutagenicity assay, in vitro human lymphocyte micronucleus assay, or an in vivo micronucleus and comet assay in mice following three daily SC doses up to $2000 \mathrm{mg} \mathrm{kg}^{-1}$ (Supplementary Table 2). In addition, no mitochondrial toxicity was observed in vitro in human cells under conditions that require mitochondrial oxidative metabolism, and in vivo where mitochondrial density and morphology were examined by transmission electron microscopy 
a
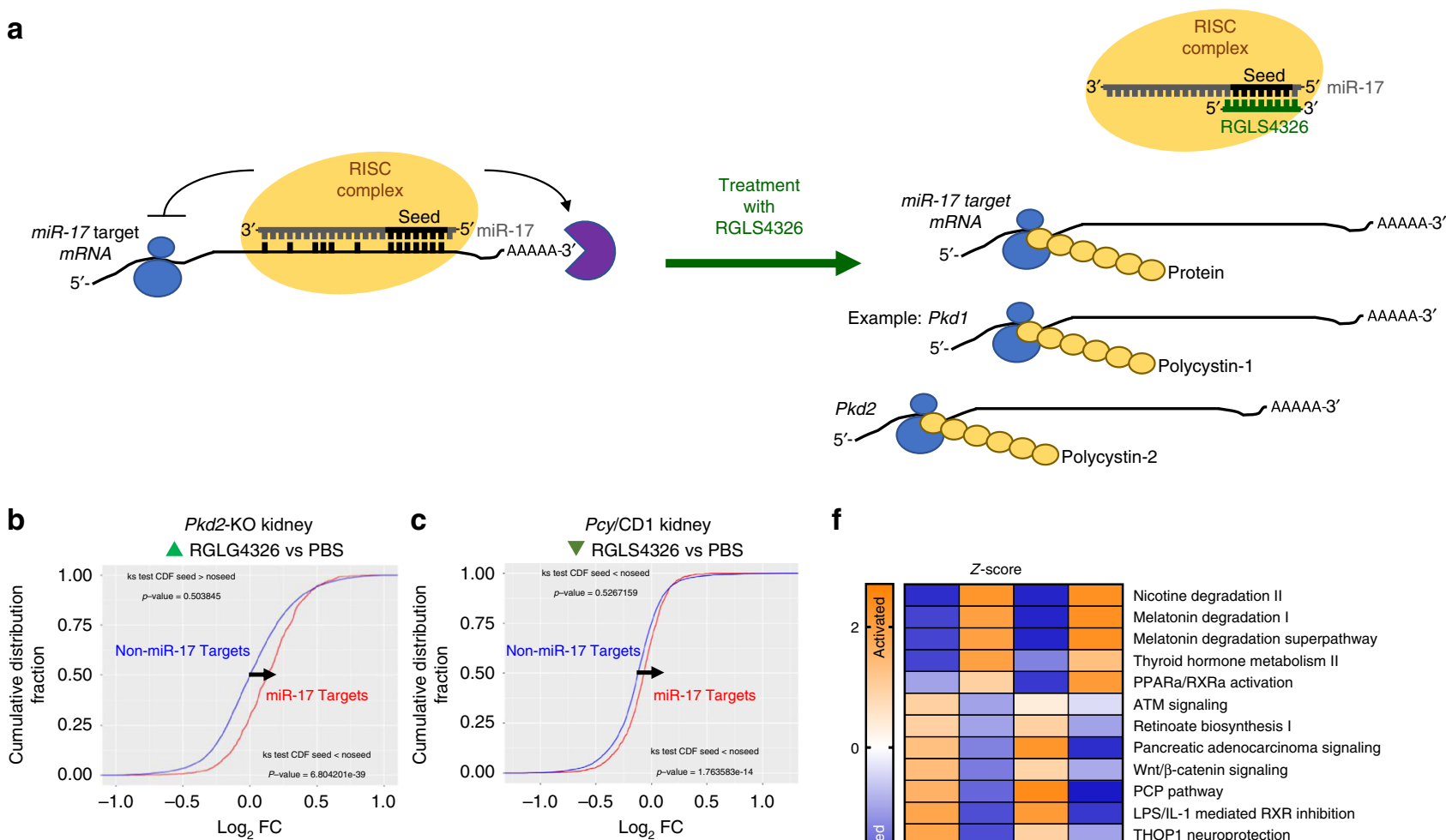

f

d

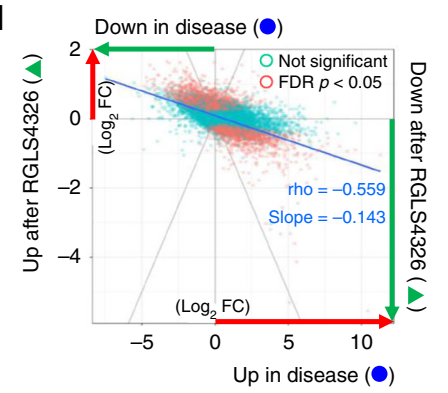

e

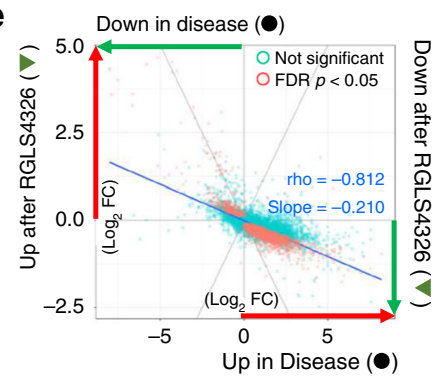

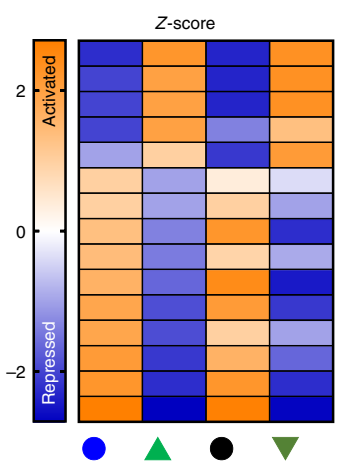

Nicotine degradation II Melatonin degradation 1 Melatonin degradation superpathway Thyroid hormone metabolism II PPARa/RXRa activation ATM signaling Retinoate biosynthesis I Pancreatic adenocarcinoma signaling Wnt/ $\beta$-catenin signaling PCP pathway LPS/L-1 mediated RXR inhibition THOP1 neuroprotection Leukocyte extravasation signaling Basal cell carcinoma signaling Colorectal cancer metastasis signaling
- Changes in Pkd2-KO vs non-transgenic $\triangle$ Changes in Pkd2-KO (RGLS4326) vs Pkd2-KO (PBS)

- Changes in Pcy/CD1 vs WT/CD1 $\nabla$ Changes in Pcy/CD1 (RGLS4326) vs Pcy/CD1 (PBS)

Fig. 7 RGLS4326 improves expression of dysregulated gene networks in PKD models. a Schematic illustration of RGLS4326-mediated inhibition of miR-17. RGLS4326 displaces miR-17 from the translationally active polysome fractions and de-represses miR-17 target genes including Pkd1 and Pkd2 and their encoded proteins PC1 and PC2. b-e RNA-seq analysis was performed to compare mRNA expression profiles between kidneys from non-transgenic ( $n=$ 12), PBS-treated Pkd2-KO ( $n=8)$, RGLS4326-treated Pkd2-KO $(n=11)$, and control oligo-treated Pkd2-KO mice $(n=8)$. RNA-seq analysis was also performed from wild-type $(n=2)$, PBS-treated Pcy/CD1 $(n=4)$, RGLS4326-treated Pcy/CD1 $(n=4)$, and control oligo-treated Pcy/CD1 mice $(n=3)$. b-c Kolmogorov-Smironov test statistics comparing the cumulative distribution of global mRNA changes between RGLS4326-treated vs. PBS-treated kidney samples indicated significant de-repression of predicted miR-17 target genes (as defined by TargetScanMouse v7.1) after RGLS4326 treatment in Pkd2-KO (green triangle) and Pcy/CD1 model (inverted green triangle). d-e Comparative differential expression analysis demonstrated a clear trend in global transcriptomic changes where dysregulated gene expression in Pkd2-KO (blue circles) and Pcy/CD1 kidneys (black circles) ( $x$-axis) were improved after RGLS4326 treatment ( $y$-axis). Rho-values and slopes from corresponding Spearman's correlations are shown. RGLS4326 treatment improves the expression of 994 genes in Pkd2-KO $(n=11)$ and 658 genes in Pcy/CD1 kidney $(n=4)$ (FDR $<0.05$ and $\log _{2}$ FC $>|0.5|$ ). $\mathbf{f}$ Top 15 pathways as predicted by the ingenuity pathway analysis software (based on $\mid z$-scores $\mid$ ) potentially responsible for the gene changes are shown. Positive $z$-scores (shades of orange) indicate activation, while negative z-scores (shades of blue) indicate repression. Source data for Fig. $7 \mathbf{f}$ is provided in Source data files

in liver and mitrochondria-rich cardiac tissues following repeat SC dosing of RGLS4326 in monkeys.

Given that certain oligonucleotides can evoke acute inflammatory response, we also evaluated the potential pro-inflammatory properties of RGLS4326 in rat tissue slice assays ex vivo and acute mouse studies in vivo. RGLS4326 was found to have low risk for pro-inflammatory effects, as no changes were observed in the well-characterized proinflammatory genes Oasla and Ifit3 following RGLS4326 treatment.

Genetic deletion of miR-17 92 in adult mice has been shown to reduce various hematopoietic cell populations ${ }^{25}$. We therefore examined the effect of RGLS4326 on these cell populations in cynomolgus monkeys and CD1 mice after repeat RGLS4326 SC administrations. Consistent with the preferential kidney distribution profile of RGLS4326, repeat dosing of RGLS4326 in monkeys or mice even at supratherapeutic ranges did not affect the various cell populations, including white blood cells, red blood cells, platelets and lymphocytes (Fig. 8a-d and Supplementary Fig. 6A-D). More importantly, although renal toxicity has been observed with certain classes of oligonucleotide therapeutics, RGLS4326 treatment had no detrimental effect on kidney function in either species (Fig. 8e-h and Supplementary Fig. $6 \mathrm{E}-\mathrm{H})$. Altogether, our data shows that RGLS4326 has a favorable preclinical safety profile. 
a

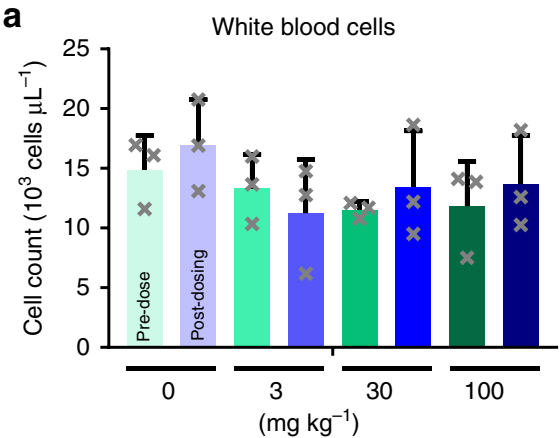

C

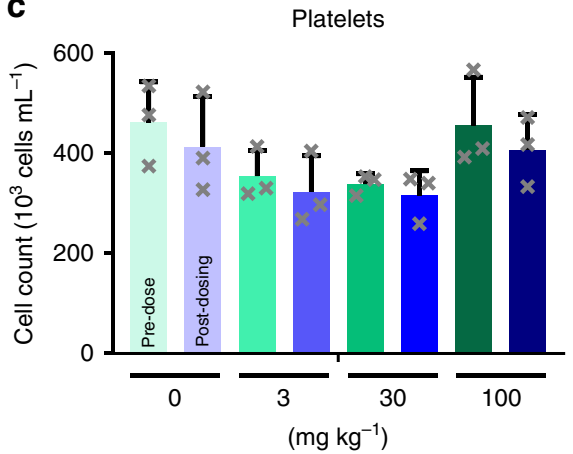

e

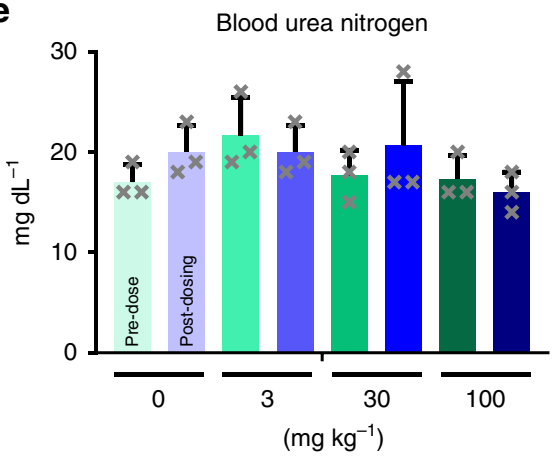

g

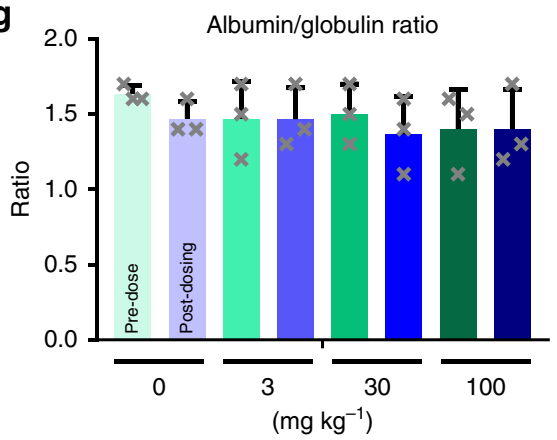

b

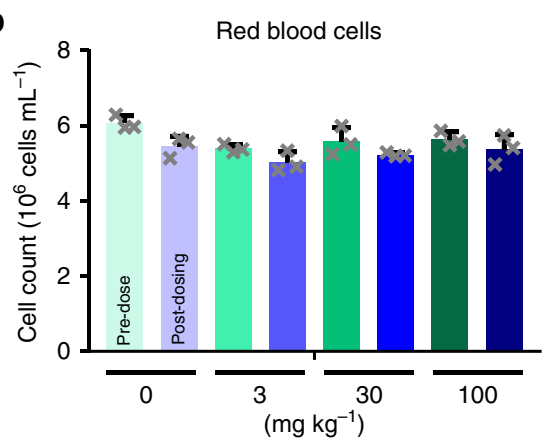

d

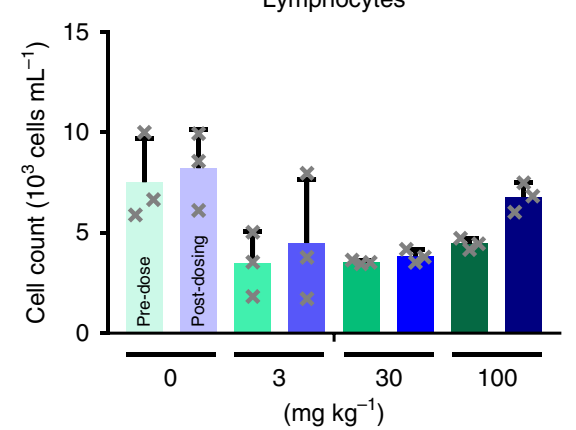

f

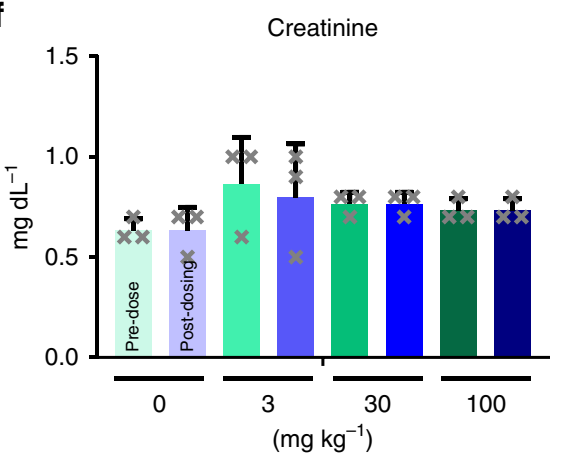

h

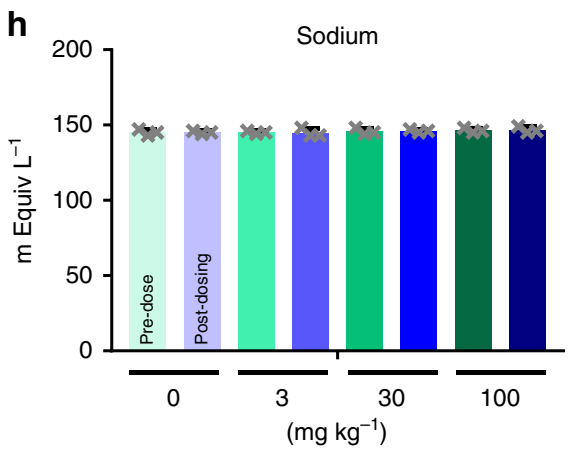

$$
\begin{array}{|ll|}
\hline \text { Male } 0 \mathrm{mg} \mathrm{kg}^{-1} \text { (Pre-dose) } & \text { Male } 0 \mathrm{mgkg}^{-1}(\text { Post-QW } \times 7) \\
\text { Male } 3 \mathrm{mg} \mathrm{kg}^{-1} \text { (Pre-dose) } & \text { Male } 3 \mathrm{mg} \mathrm{kg}^{-1}(\text { Post-QW } \times 7) \\
\text { Male } 30 \mathrm{mg} \mathrm{kg}^{-1} \text { (Pre-dose) } & \text { Male } 30 \mathrm{mg} \mathrm{kg}^{-1}(\text { Post-QW } \times 7) \\
\text { Male } 100 \mathrm{mg} \mathrm{kg}^{-1} \text { (Pre-dose) } & \text { Male } 100 \mathrm{mg} \mathrm{kg}^{-1}(\text { Post-QW } \times 7) \\
\hline
\end{array}
$$

Fig. 8 RGLS4326 does not cause hematopoietic or kidney toxicity in monkeys. Male cynomolgus monkeys ( $n=3 /$ group) were dosed SC QW with 0, 3, 30, or $100 \mathrm{mg} \mathrm{kg}^{-1}$ of RGLS4326 for 7 consecutive weeks (QW 7 ). Prior to the study, animals were acclimated to the study room for 14 days. On the final day of acclimation, animals weighed between 2.8 and $6.1 \mathrm{~kg}$ and were 3 to 6 years of age. Blood was collected for hematology and serum chemistry analysis before and at $48 \mathrm{~h}$ after the last dose. Specimens were analyzed using an Advia 120 automated hematology analyzer and an AU680 chemistry analyzer. Comparison between pre-dose and post-QWx7 values for each treatment groups indicated no hematological abnormalities (a-d) and no renal toxicity (e-h) following RGLS4326 treatment. Error bars represent standard deviations. Source data for Fig. 8a-h is provided in Source data files 


\section{Discussion}

Chronic kidney diseases are of significant societal burden on par with cancer and cardiovascular diseases; however, drug development in this area, including ADPKD, has been limited. Here, we report the discovery and preclinical evaluation of RGLS4326 as a potential disease-modifying treatment for ADPKD.

Several key attributes make RGLS4326 an attractive potential therapeutic agent for ADPKD. First and foremost, RGLS4326 is efficacious in multiple PKD mouse models across a range of doses and treatment regimens. The magnitude of effectiveness as determined by MRI-based TKV (50\% reduction in bwTKV) is comparable to that observed for tolvaptan treatment in the TEMPO 3:4 clinical trial (49\% improvement in yearly TKV) ${ }^{33}$. Considering that RGLS4326 and tolvaptan function through independent mechanisms, combining these two distinct drug classes may produce synergistic beneficial effects and additional investigations are warranted.

Secondly, we provide several lines of evidence that RGLS4326 preferentially distributes to kidney and renal tubules, thereby minimizing exposure and potential toxicity to other cells and tissues where miR-17 might also play important roles, such as hematopoietic cells. While the mechanisms for the differential exposure are not entirely clear, our data showed that RGLS4326 was $>8$-fold more abundant in kidney compared to liver following SC administration, perhaps due to its short 9-nt length compared to other oligonucleotide therapeutics. The QWBA data also showed that RGLS4326 has very low exposure in other tissues such as adrenal gland, spleen, bone marrow, and lymph nodes. Indeed, we observed no hematological abnormalities after RGLS4326 treatment in both mice and monkeys, even at very high supratherapeutic doses.

Thirdly, RGLS4326 remains active in the kidney for at least 14 days. In contrast, tolvaptan, as well as other medications that are currently in clinical trials for ADPKD, are administered once or twice daily. Indeed, we have demonstrated that RGLS4326 treatment, ranging from weekly and up to monthly administrations, confers efficacy in different mouse models of PKD. The longer half-life in kidney, which allows for less frequent dosing, is a desirable feature for chronic diseases like ADPKD that require long-term treatment.

miRNA-based drugs have a unique MOA compared to traditional drugs. miRNAs simultaneously repress many mRNA targets, thereby affecting multiple signaling pathways. Moreover, miRNAs function as rheostats rather than on-off switches. Whereas traditional drugs often inhibit (or activate) a single or few pathways, a miRNA-based therapeutic can comprehensively modulate multiple pathways. Consistent with this idea, we showed that RGLS4326 treatment improves the expression of many pathogenic gene networks and pathways in PKD. Importantly, this coordinated rewiring of the polycystic kidney transcriptome towards a normal kidney profile exemplifies the potential of RGLS4326 as a disease-modifying treatment for ADPKD. Finally, one of the most intriguing features of RGLS4326 is its potential direct mechanistic link to ADPKD. We and others have shown that miR-17 binds to the $3^{\prime}$ UTRs of PKD1 and PKD2 and inhibits their expression ${ }^{18,38,39}$. Accordingly, RGLS4326 promotes the expression of both genes in murine and human kidney cells. It is therefore tempting to speculate that part of the RGLS4326 therapeutic efficacy is via direct $P K D 1 / 2$ derepression. This MOA is especially of relevance in the context of hypomorphic mutations, where RGLS4326-mediated increase in $P K D 1 / 2$ gene dosage above a critical threshold may alleviate $\mathrm{ADPKD}^{2}$

In conclusion, we have discovered a first-in-class anti-miR-17 oligonucleotide RGLS4326 that has promising potential as drug treatment for ADPKD. RGLS4326 has favorable potency, stability, safety, pharmacokinetic, and pharmacodynamic characteristics, including preferential distribution to kidney. RGLS4326 attenuates cyst growth in human ADPKD models in vitro and is efficacious in multiple PKD mouse models in vivo. Our data strongly support the clinical development of RGLS4326 for ADPKD.

\section{Methods}

Cell lines. Mouse IMCD3 kidney collecting duct cells (CRL-2123; ATCC) were cultured with 1:1 DMEM/F-12 medium (ATCC) supplemented with 10\% FBS and $1 \%$ penicillin-streptomycin. Mouse M1 kidney collecting duct cells (ATCC CRL2038) were cultured with 1:1 DMEM/F12 supplemented with $2.5 \mathrm{mM}$ L-glutamine, $15 \mathrm{mM}$ HEPES, $0.5 \mathrm{mM}$ sodium pyruvate, $1.2 \mathrm{~g} \mathrm{~L}^{-1}$ sodium bicarbonate, $0.005 \mathrm{mM}$ dexamethasone, and 5\% FBS. Mouse DBA-WT and DBA-PKD kidney collecting duct cells and mouse LTL-WT and LTL-PKD kidney proximal tubular cells (Discovery BioMed, Inc) were cultured with 1:1 RenaLife Complete Medium (Lifeline Cell Technology) and Advance MEM medium (Invitrogen) supplemented with $5 \%$ FBS, $4 \mathrm{mM}$ L-Glutamine and $1 \%$ of penicillin-streptomycin ${ }^{40}$. Mouse Myc-driven HCC cell line obtained from Dr. Dean Felsher Lab were cultured in DMEM (Invitrogen) supplemented with $10 \% \mathrm{FBS}^{41}$. Mouse MDCT kidney distal convoluted tubule cells (ATCC CRL-3250) were cultured in 1:1 DMEM/F-12 supplemented with $5 \% \mathrm{FBS}, 1 \mathrm{~g} \mathrm{~L}^{-1}$ glucose, and $1 \mathrm{mM}$ sodium pyruvate. Mouse NIH/ 3T3 (ATCC CRL-1658), Hep-C7 (ATCC CRL-2026), and HeLa (ATCC CCL-2) cells were cultured according to ATCC's protocols. All cells were incubated in an atmosphere of $95 \%$ air and $5 \% \mathrm{CO}_{2}$ at $37^{\circ} \mathrm{C}$

Ex-vivo tissue slice assay. Two hundred micro $(\mu \mathrm{m})$ thin precision-cut tissue slices were freshly prepared from kidneys of 8-week-old WT/C57BL6 mice, or kidneys and livers from 8-week-old Sprague-Dawley rats using Brendel/Vitron tissue slicer under cold oxygenated preservation solution V7 (Vitron) and incubated in Waymouth's medium (Gibco) supplemented with 10\% FBS and 1\% Antimycotic at $37^{\circ} \mathrm{C}, 95 \% \mathrm{O}_{2}, 5 \% \mathrm{CO}_{2}$ on a rotating Vitron incubator. Two hours post-slicing, the medium was replaced with fresh Waymouth's medium containing RGLS4326, oligo control or PBS, and replenished every $24 \mathrm{~h}$. For pro-inflammatory liability assessment, rat liver and kidney slices were incubated with $5 \mu \mathrm{M}$ RGLS4326 for $24 \mathrm{~h}$ and $48 \mathrm{~h}$, respectively, prior to RNA extraction and analysis. For miR-17 PD-Sig analysis, mouse kidney slices were incubated at $10 \mu \mathrm{M}$ RGLS4326 for $72 \mathrm{~h}$

Primary human ADPKD cyst assay. Primary human ADPKD cyst cells were obtained from PKD Research Biomarker and Biomaterial Core at the University of Kansus Medical Center (KUMC). Informed consent was obtained from all the human participants and the protocol for the use of surgically discarded kidney tissues complied with federal regulations and was approved by the Institutional Review Board at the University of Kansas Medical Center. Cells were cultured in DMEM/F12++(cat \# 10565-018, Gibco) supplemented with 5\% FBS, $5 \mu \mathrm{g} \mathrm{kg}^{-1}$ insulin, $5 \mu \mathrm{g} \mathrm{mL}^{-1}$ transferrin and $5 \mathrm{ng} \mathrm{mL}^{-1}$ sodium selenite and incubated in an atmosphere of $95 \%$ air and $5 \% \mathrm{CO} 2$ at $37^{\circ} \mathrm{C}$ until $80 \%$ confluency. Tryptinized cells $(100,000$ cells/well in a 6 well plate) were reverse transfected with Lipofectamine RNAiMAX reagent (cat \# 13778-150, Life Technologies) with RGLS4326, oligo control or PBS. Twenty four hours later, cells were tryptinized, counted and plated at 4000 cells/well density in $130 \mu \mathrm{l}$ of media plus Matrigel (cat \# 354234 , Corning) in a 96-well plate (cat \# 353072, Corning). The gel was allowed to solidify before $130 \mu \mathrm{l}$ of transfection media $(117 \mu \mathrm{l}$ of complete media and $13 \mu \mathrm{l}$ of transfection complex) was added to the top of the matrigel. Media was replenished every $72 \mathrm{~h}$ until 8 days post plating when the cyst number and volume were assessed. Images (twenty-eight 24-bit colour TIFF images at 2448-by-1920 pixels at 72 dpi) from each well were captured with an Olympus DP26 camera (Olympus Corporation) and were comprised of 28 images "stacks" taken 150 microns apart. Image processing took place using a custom script written in the $\mathrm{R}$ language employing the EBImage Bioconductor image processing library ${ }^{42}$. Images from one well were processed as a batch, with each image from each image stack undergoing segmentation analysis to detect objects in each image of a different contrast. Per detected object, the following statistics were collected: mean radius in pixels, CV of the radius, and the eccentricity (i.e., deviation from circularity where a perfect circle $=0$ ). Images were filtered to remove noise and non-cyst objects by removing objects less than 15 and greater than 200 pixel mean radius, greater than $0.2 \mathrm{CV}$ radius and greater than 0.75 eccentricity. Images were considered in the context of the entire well because larger cysts span more than one image in the image stack. Cyst objects detected in the same location in sequential images were considered to be the same cyst. Assuming the cyst was spherical in shape, the largest cyst object in the series of objects was chosen, its radius ( $r$ ) was found and the volume of the cyst was calculated using the formula $4 / 3 \pi \mathrm{r}^{3}$. This same exercise was performed using all wells (i.e., all image stacks) for all donors.

Animals. $P k d 2-\mathrm{KO}$, JCK/C57BL6 (C57Bl/6J-nek8 jck, Crown Bio), Pcy/CD1 (CD-1pcylusm, Crown Bio), Pcy/DBA (DBA/2FG-pcy, Kyudo Co), $129 \times 1 /$ SvJ (Jackson laboratory), WT/C57BL6 mice (C57BL/6J, Jackson laboratory), WT/DBA (Charles River), CD1 mice (Charles River and SNBL-USA), Sprague-Dawley rats (Charles 
River), and Cynomolgus Monkey (SNBL-USA) were used in this study. All animal experiments were conducted in accordance with the Institutional AAALAC Guidelines and approved by the Institutional Animal Care and Use Committees at Explora, UT Southwestern, InVicro, and SNBL-USA where the experiments were performed.

Luciferase sensor assay. HeLa cells were transfected for $4 \mathrm{~h}$ with Synthetic miRNA Target GoClone Reporters (harboring two fully complementary miRNA binding sequence of interest at $3^{\prime}$ UTR of the luciferase gene; Active Motif) and Human pre-microRNA Expression Construct Lenti-miRNAs of interest (System Biosciences) using Lipofectamine LTX-Plus reagent (Life Technologies). Cells were seeded overnight prior to transfection with anti-miR for $24 \mathrm{~h}$ using Lipofectamine RNAiMAX (Life Technologies). Luciferase activity was measured using LightSwitch Luciferase assay kit (Active Motif). Raw luciferase data was background subtracted and normalized to untreated control. $\mathrm{EC}_{50}$ values were estimated using GraphPad Prism software v7.04.

miRNA expression analysis. Reverse transcription was performed using the $\mathrm{TaqMan}^{\circledR}$ MicroRNA reverse transcription kit (Applied Biosystem) and TaqMan ${ }^{\circledR}$ miRNA assays (Applied Biosystem). Real-time PCR was performed using TaqMan ${ }^{\circledR}$ Universal PCR Master Mix II according to the manufacturer's protocol. List of TaqMan ${ }^{\circledast}$ miRNA Assays used is shown in Supplementary Table 3.

Gene expression analysis. Total RNA was isolated using the RNeasy mini kit (Qiagen). Reverse transcription and preamplification reactions were performed using Bio-Rad T100 Thermocycler (Bio-Rad) according to Fluidigm's protocol. Quantitative real-time PCR was performed using the BioMark Real-Time PCR System Dynamic Array 192.24 IFC (Fluidigm) according to the manufacturer's protocols. Data were analyzed using BioMark Real-Time PCR analysis software version 2 (Fluidigm). Relative gene expression values were determined using the $\Delta \Delta C T$ method. The mouse miR-17-PD-Sig was calculated as the average log2 fold change in expression of 18 mouse miR-17 target genes (Plekha3, Rhoc,

D030056l22rik, Clock, Polq, Mtf1, E2f1, Tgfbr2, P2rx4, Nagk, Mink1, Zfp367, Fyco1, $P f k p, D d h d 1, C d c 37 l 1, W f_{s} 1$, and St6galnac6) normalized by 6 housekeeping genes (Tbp, Gusb, Rplp0, Hprt1, B2m, and Paklip1) compared to Mock (transfection) or PBS (free-uptake) for in vitro studies, while a representative 10 mouse genes (Plekha3, Clock, Polq, Mtf1, Nagk, Mink1, Zfp367, Fyco1, Ddhd1, and Cdc37l1) was used for in vivo Pkd2KO mouse studies. The human miR-17 PD-Sig ${ }^{27}$ was calculated as average log2-fold change in expression of 13 human miR-17 target genes (AMPD3, BTG3, C7ORF43, CROT, ENPP5, LIMK1, MINK1, NAGK, NKIRAS1, PLEKHA3, PTPN4, TBC1D9, and TGFBR2) normalized by 6 housekeeping genes (B2M, GUSB, HPRT1, RPLPO, TBP, and UBC). The list of probes used is shown in Supplementary Table 4 .

RNA-sequencing analysis. RNA-Seq libraries were prepared using TruSeq stranded mRNA LT sample prep kit and ran on NextSeq 500 sequencer per manufacturer's protocol (Illumina). Sequencing quality was assessed with the FastQC tool and trimmed with TrimGalore. Samples between $2 \times 10^{7}$ and $6 \times 10^{7}$ paired-end and 75 bps long reads were mapped with STAR on Mus musculus (GCA000001635.2) genome assembly GRCm38 (mm10) from Genome Reference Consortium. Uniquely mapped reads were counted in genes using HTseq, while mapping and counting quality were assessed by Picard (http://broadinstitute. github.io/picard/) and RSeQC tool. Differential expression was performed using edgeR v3.20.2. Kolmogorov-Smironov test statistics comparing cumulative distribution fraction (CDF) of global mRNA changes for predicted miRNA targets based on TargetScanMouse v7.1 or TargetScanHuman v7.1 were assessed ${ }^{43}$. Ingenuity Pathways Analysis software was used to predict putative gene networks involved with input differential mRNA expression data.

Quantification of RGLS4326 in plasma and tissues. Plasma ( $0-8 \mathrm{~h}$ post dose) and tissue concentrations of RGLS4326 were measured using high-performance liquid chromatography time-of-flight mass spectrometry (HPLC-TOF). Plasma samples from 24-1344 h post dose were quantified using high-performance liquid chromatography with fluorescence detection (HPLC-FL). For HPLC-TOF, RGLS4326 was isolated from $100 \mu \mathrm{L}$ of plasma or $20 \mathrm{mg}$ of tissue by liquid-liquid extraction followed by solid-phase extraction steps. For HPLC-FL, $30 \mu \mathrm{L}$ plasma was subjected to proteinase digestion followed by precipitation and hybridization of the supernatant to fluorescent probe complementary to RGLS4326, prior to injection into fluorescence detector-equipped HPLC. Calibration curve samples were prepared by spiking blank plasma or tissue homogenates with known concentrations of RGLS4326 and co-extracting calibration-samples alongside with samples. Mass spectrometry or fluorescent signal from samples were extrapolated to the calibration curve to obtain concentration measurements. Signal analysis was performed using MassHunter Version 7.0 (Agilent Technologies). A value of zero was assigned to concentrations below the limit of quantification. Pharmacokinetic parameters were determined using Phenoix ${ }^{\circledast}$ WinNonlin ${ }^{\circledR}$ version 6.3 (Certara L.P. (Pharsight), St. Louis, MO).
Western blot analysis. Cells were lyzed in PIERCE RIPA buffer (ThermoFisher Scientific). A total of $20 \mu \mathrm{g}$ of protein were loaded on a NuPAGE 3-8\% TrisAcetate gel (Invitrogen), transferred to $0.45 \mu \mathrm{m}$ PVDF membranes, immunoblotted with anti-PC1 (1:200, 7E12, sc-130554, SantaCruz), anti-PC2 (1:200, D-3, sc-28331, SantaCruz) or anti-ActinB (1:5000, BA3R, Invitrogen) antibodies, and detected with chemiluminescence using standard protocols. Original western blot images are shown in Supplementary Fig. 7.

Histology, cyst index, and immunofluorescence staining. $P k d 2$-KO left kidney was perfused with cold PBS and 4\% PFA prior to collection. All other mouse kidneys were collected, fixed in $10 \%$ formalin, dehydrated, and embedded in paraffin using a standard protocol. Samples were sectioned at $5 \mu \mathrm{m}$ and subjected to hematoxylin and eosin staining. Cyst index (cystic area/total kidney section surface area) was performed using ImageJ analysis software. For immunofluorescence staining, the following antibodies and dilutions were used: anti-PS (Regulus Therapeutics Inc, 1:1000), anti-phosphohistone H3 (1:400, Sigma-Aldrich H0412), Biotinylated Dolichos biflorus agglutinin (DBA; Vector Laboratories, 1:500), and Fluorescein Lotus tetragonolobus agglutinin (LTA; Vector Laboratories, 1:200). Secondary antibodies were conjugated to Alexa Fluor 488 or Alexa Fluor 594 (Molecular Probes, 1:400).

Quantification of urinary Ngal. Enzyme-linked immunosorbent assay (ELISA) was performed on mouse urine samples (diluted 1:200) with Mouse Lipocalin-2/ NGAL Quantikine ELISA kit (R\&D Systems) according to the manufacturers' instructions. Values were normalized with urine creatinine (UCr) level and expressed as $\mu \mathrm{g} \mathrm{Ngal}$ per mg UCr.

Quantitative whole-body autoradiography (QWBA). Tissue distribution was assessed based on QWBA of [ $\left.{ }^{35} \mathrm{~S}\right]$-RGLS4326-derived radioactivity in male CD-1 mice following a single subcutaneous (SC) dose of RGLS4326 at $30 \mathrm{mg} \mathrm{kg}^{-1}$ and a target radioactivity of $100 \mu \mathrm{Ci} \mathrm{kg}^{-1}$. At approximately 48 and $672 \mathrm{~h}$ post dose, mice were euthanized, and carcasses were submerged in a dry ice/hexane bath. The frozen carcasses were individually set in a mold, submerged in $5 \%\left(\mathrm{w} \mathrm{v}^{-1}\right)$ low viscosity carboxymethylcellulose (CMC), and embedded by placing the stage (mold) in a dry ice/hexane bath. Frozen blocked carcasses were removed from dry ice/hexane bath, and 1/4-inch holes were drilled into the sample blocks and filled with calibration standards containing known quantities of $\left.{ }^{35} \mathrm{~S}\right]$-RGLS4326, prior to sectioning. Thirty microns thick sagittal sections of the CMC-embedded mouse carcasses, including calibration standards were sectioned and imaged using phosphor imaging plates. Autoradioluminograms of the sections were compared to calibration standards to obtain a measurement of the concentration of $\left[{ }^{35} \mathrm{~S}\right]-$ RGLS4326.

Efficacy studies. For $P k d 2$-KO mouse model, mice were randomly assigned and administered by SC injection with phosphate-buffered saline (PBS), $20 \mathrm{mg} \mathrm{kg}^{-1}$ of RGLS4326 or oligo control on post-natal day (P)10, P11, P12, and P19. All animals were euthanized, and samples harvested at P13, P16, P19, and P28. Non-transgenic strain-matched mice were also sacrificed on same days. Each group had 5-12 mice. Investigators were blinded to treatment groups until predetermined analysis was completed. For $P c y / C D 1$ mouse model, 5-weeks old mice were randomly assigned and dosed with RGLS4326 or control oligo at $25 \mathrm{mg} \mathrm{kg}^{-1}$ or PBS by SC injections once-weekly (QW) or monthly (Q4W) for 25 consecutive weeks. An additional group of 15-weeks-old Pcy/CD1 mice were dosed with $25 \mathrm{mg} \mathrm{kg}^{-1}$ RGLS4326 SC QW for 15 consecutive weeks. Five untreated male WT/CD1 mice were included for comparison. All animals were euthanized, and samples harvested at 30 weeks of age (7 days after the last dose). Each group had 15 mice. For $P c y /$ DBA mouse model, 6-weeks old male mice were randomly assigned and dosed SC with PBS, control oligonucleotide at $25 \mathrm{mg} \mathrm{kg}^{-1}$, or RGLS4326 at 1, 5, or $25 \mathrm{mg} \mathrm{kg}^{-1} \mathrm{QW}$ or with RGLS4326 at $25 \mathrm{mg} \mathrm{kg}^{-1} \mathrm{Q} 4 \mathrm{~W}$ for 9 consecutive weeks. An additional group of mice were treated with $0.3 \%$ tolvaptan via diet from 6 to 14 weeks of age. Five male WT/DBA mice treated with PBS SC QW were included for comparison. All animals were euthanized, and samples harvested at 15 weeks of age ( 7 days after the last dose). Each group had 15 mice. For magnetic-resonance imaging (MRI) study, male $P c y / \mathrm{DBA}$ mice were imaged at baseline at 6 weeks of age prior to treatment group assignment. All mice were dosed SC QW with PBS $(n=5)$ or $30 \mathrm{mg} \mathrm{kg}^{-1}$ RGLS4326 $(n=10)$ starting at 7 weeks of age for 8 consecutive weeks. Additional MRI was performed at 8,11 , and 14 weeks of age.

Magnetic resonance imaging (MRI) protocol. MRI scanning was accomplished using an 11.7T Bruker Biospec/Avance III system and a $38 \mathrm{~mm}$ volume RF coil. After induction of anesthesia, mice were placed inside the coil with respiratory monitoring, and warm air heating was used throughout. After scout scans for slice planning, a multi-slice T2-weighted RARE protocol (repetition time $=1.2 \mathrm{~s}$; RARE factor $=8$; effective echo time $=22.5 \mathrm{~ms}$ ), was used with $0.75 \mathrm{~mm}$ thick contiguous coronal slices used to cover the extent of both kidneys. The field of view was $30 \times$ $30 \mathrm{~mm}$ over a $256 \times 256$ matrix. 
In vivo acute pro-inflammatory liability mouse study. Male 6-10 weeks-old $129 \times 1 /$ SvJ mice were administered a single SC dose of RGLS4326 at $300 \mathrm{mg} \mathrm{kg}^{-1}$ or PBS and euthanized at $96 \mathrm{~h}$. Kidney and liver samples were harvested and snapfrozen for RNA extraction. Relative gene expression of Oasl2 and Ifit1 in kidney and liver were determined using the $\Delta \Delta \mathrm{CT}$ method compared to PBS. Clinical chemistry was assessed by the Axcel Blood Chemistry Analyzer (Alfa Wassermann, Caldwell, NJ).

Reporting summary. Further information on research design is available in the Nature Research Reporting Summary linked to this article.

\section{Data availability}

The RNA-seq data has been deposited in the NCBI Gene Expression Omnibus repository under accession number GSE134721. The source data underlyging Figs. 1b-f, 2a-f, 3b-e, $\mathrm{g}-\mathrm{h}, 4 \mathrm{a}-\mathrm{c}, \mathrm{f}-\mathrm{i}, 5 \mathrm{a}-\mathrm{c}, \mathrm{e}-\mathrm{f}, \mathrm{h}-\mathrm{k}, 6 \mathrm{~b}-\mathrm{c}, \mathrm{e}, 7 \mathrm{f}$, and $8 \mathrm{a}-\mathrm{h}$, as well as Supplementary Figs 1A-E, $2 \mathrm{C}-\mathrm{D}, 3 \mathrm{~A}-\mathrm{D}$, and $6 \mathrm{~A}-\mathrm{H}$, are provided as a Source Data File.

Received: 10 January 2019 Accepted: 9 August 2019

Published online: 12 September 2019

\section{References}

1. Torres, V. E. \& Harris, P. C. Autosomal dominant polycystic kidney disease: the last 3 years. Kidney Int. 76, 149-168 (2009).

2. Harris, P. C. \& Torres, V. E. Genetic mechanisms and signaling pathways in autosomal dominant polycystic kidney disease. J. Clin. Invest. 124, 2315-2324 (2014).

3. Chapman, A. B. et al. Autosomal-dominant polycystic kidney disease (ADPKD): executive summary from a Kidney Disease: Improving Global Outcomes (KDIGO) Controversies Conference. Kidney Int. 88, 17-27 (2015).

4. Baek, D. et al. The impact of microRNAs on protein output. Nature 455, 64-71 (2008).

5. Bartel, D. P. MicroRNAs: genomics, biogenesis, mechanism, and function. Cell 116, 281-297 (2004)

6. Bartel, D. P. MicroRNAs: target recognition and regulatory functions. Cell 136, 215-233 (2009).

7. Carney, E. F. Polycystic kidney disease: MicroRNA-17: a new drug target for ADPKD. Nat. Rev. Nephrol. 13, 260 (2017).

8. Krutzfeldt, J. et al. Silencing of microRNAs in vivo with 'antagomirs'. Nature 438, 685-689 (2005).

9. Mendell, J. T. \& Olson, E. N. MicroRNAs in stress signaling and human disease. Cell 148, 1172-1187 (2012).

10. Rupaimoole, R. \& Slack, F. J. MicroRNA therapeutics: towards a new era for the management of cancer and other diseases. Nat. Rev. Drug Disco. 16, 203-222 (2017).

11. Chau, B. N. et al. MicroRNA-21 promotes fibrosis of the kidney by silencing metabolic pathways. Sci. Transl. Med. 4, 121 ra118 (2012).

12. Gomez, I. G. et al. Anti-microRNA-21 oligonucleotides prevent Alport nephropathy progression by stimulating metabolic pathways. J. Clin. Invest. 125, 141-156 (2015).

13. Janssen, H. L. et al. Treatment of HCV infection by targeting microRNA. $N$. Engl. J. Med. 368, 1685-1694 (2013).

14. Lakhia, R. et al. MicroRNA-21 aggravates cyst growth in a model of polycystic kidney disease. J. Am. Soc. Nephrol. 27, 2319-2330 (2016).

15. Wagenaar, T. R. et al. Anti-miR-21 suppresses hepatocellular carcinoma growth via broad transcriptional network deregulation. Mol. Cancer Res. 13, 1009-1021 (2015).

16. van der Ree, M. H. et al. Safety, tolerability, and antiviral effect of RG-101 in patients with chronic hepatitis $\mathrm{C}$ : a phase $1 \mathrm{~B}$, double-blind, randomised controlled trial. Lancet 389, 709-717 (2017).

17. Hajarnis, S. et al. microRNA-17 family promotes polycystic kidney disease progression through modulation of mitochondrial metabolism. Nat. Commun. 8, 14395 (2017).

18. Patel, V. et al. miR-17 92 miRNA cluster promotes kidney cyst growth in polycystic kidney disease. Proc. Natl Acad. Sci. USA 110, 10765-10770 (2013).

19. Yheskel, M., Lakhia, R., Cobo-Stark, P., Flaten, A. \& Patel, V. Anti-microRNA screen uncovers miR-17 family within miR-17 92 cluster as the primary driver of kidney cyst growth. Sci. Rep. 9, 1920 (2019).

20. Concepcion, C. P., Bonetti, C. \& Ventura, A. The microRNA-17-92 family of microRNA clusters in development and disease. Cancer J. 18, 262-267 (2012).

21. Esquela-Kerscher, A. \& Slack, F. J. Oncomirs-microRNAs with a role in cancer. Nat. Rev. Cancer 6, 259-269 (2006).

22. Mogilyansky, E. \& Rigoutsos, I. The miR-17/92 cluster: a comprehensive update on its genomics, genetics, functions and increasingly important and numerous roles in health and disease. Cell Death Differ. 20, 1603-1614 (2013).
23. Ventura, A. et al. Targeted deletion reveals essential and overlapping functions of the miR-17 through 92 family of miRNA clusters. Cell 132, 875-886 (2008)

24. Marrone, A. K. et al. MicroRNA-17 92 is required for nephrogenesis and renal function. J. Am. Soc. Nephrol. 25, 1440-1452 (2014).

25. Brinkmann, K., et al. miR17 92 is essential for the survival of hematopoietic stem and progenitor cells by restraining pro-apoptotic BIM. Preprint at bioRxviv: doi.org/10.1101/342071 (2018).

26. Androsavich, J. R. et al. Polysome shift assay for direct measurement of miRNA inhibition by anti-miRNA drugs. Nucleic Acids Res. 44, e13 (2016).

27. Huang, X. et al. Lipid nanoparticle-mediated delivery of Anti-miR-17 family oligonucleotide suppresses hepatocellular carcinoma growth. Mol. Cancer Ther. 16, 905-913 (2017).

28. Obad, S. et al. Silencing of microRNA families by seed-targeting tiny LNAs. Nat. Genet 43, 371-378 (2011).

29. Rifai, A., Brysch, W., Fadden, K., Clark, J. \& Schlingensiepen, K. H. Clearance kinetics, biodistribution, and organ saturability of phosphorothioate oligodeoxynucleotides in mice. Am. J. Pathol. 149, 717-725 (1996).

30. Masarjian, L., de Peyster, A., Levin, A. A. \& Monteith, D. K. Distribution and excretion of a phosphorothioate oligonucleotide in rats with experimentally induced renal injury. Oligonucleotides 14, 299-310 (2004).

31. Aihara, M. et al. Tolvaptan delays the onset of end-stage renal disease in a polycystic kidney disease model by suppressing increases in kidney volume and renal injury. J. Pharm. Exp. Ther. 349, 258-267 (2014).

32. Irazabal, M. V. et al. Imaging classification of autosomal dominant polycystic kidney disease: a simple model for selecting patients for clinical trials. J. Am. Soc. Nephrol. 26, 160-172 (2015).

33. Torres, V. E. et al. Tolvaptan in patients with autosomal dominant polycystic kidney disease. N. Engl. J. Med. 367, 2407-2418 (2012).

34. Lakhia, R. et al. PPARalpha agonist fenofibrate enhances fatty acid betaoxidation and attenuates polycystic kidney and liver disease in mice. Am. J. Physiol. Ren. Physiol. 314, F122-F131 (2018).

35. Janas, M. M. et al. Impact of oligonucleotide structure, chemistry, and delivery method on in vitro cytotoxicity. Nucleic Acid Ther. 27, 11-22 (2017).

36. Johnson, A. A. et al. Toxicity of antiviral nucleoside analogs and the human mitochondrial DNA polymerase. J. Biol. Chem. 276, 40847-40857 (2001).

37. Shen, W., Liang, X. H., Sun, H. \& Crooke, S. T. 2'-Fluoro-modified phosphorothioate oligonucleotide can cause rapid degradation of P54nrb and PSF. Nucleic Acids Res. 43, 4569-4578 (2015).

38. Sun, H. et al. MicroRNA-17 post-transcriptionally regulates polycystic kidney disease-2 gene and promotes cell proliferation. Mol. Biol. Rep. 37, 2951-2958 (2010).

39. Tran, U. et al. The RNA-binding protein bicaudal $\mathrm{C}$ regulates polycystin 2 in the kidney by antagonizing miR-17 activity. Development 137, 1107-1116 (2010).

40. $\mathrm{Yu}, \mathrm{S}$. et al. Essential role of cleavage of Polycystin-1 at G protein-coupled receptor proteolytic site for kidney tubular structure. Proc. Natl Acad. Sci. USA 104, 18688-18693 (2007).

41. Dhanasekaran, R. et al. Anti-miR-17 therapy delays tumorigenesis in MYCdriven hepatocellular carcinoma (HCC). Oncotarget 9, 5517-5528 (2018).

42. Pau, G., Fuchs, F., Sklyar, O., Boutros, M. \& Huber, W. EBImage-an R package for image processing with applications to cellular phenotypes. Bioinformatics 26, 979-981 (2010).

43. Agarwal, V., Bell, G. W., Nam, J. W. \& Bartel, D. P. Predicting effective microRNA target sites in mammalian mRNAs. Elife 4, e05005 (2015).

\section{Acknowledgements}

We thank Regulus Therapeutics employees and members of the Patel Lab for their support of this project. We thank Vincente Torres and Peter Harris for guidance at the initial stage of this work. We thank Darren Wallace and the PKD biomarkers and Biomaterials Core of the University of Kansas Medical Center for providing the human primary ADPKD cyst cells. We thank Stefan Somolo for providing $P k d 2^{\mathrm{F} / \mathrm{F}}$ mice. We thank the University of Texas Southwestern O’Brien Kidney Research Core center for providing critical reagents and services. We thank InVicro, a Konica Minolta Company for providing services for MRI-imaging. The Patel lab is supported by grants from the National Institute of Health (R01DK102572) and the Department of Defense (D01 W81XWH1810673). This work is funded by Regulus Therapeutics.

\section{Author contributions}

E.C.L. and C.A. led the scientific team and designed research; E.C.L., T.V., C.A., A.S., A.F., M.Y., K.K., J.L., M.K., T.C., R.S., S.D., J.R.A., and J.K. designed and performed experiments; E.C.L., T.V., A.S., A.F., S.G., M.T., S.L., A.P., S.S., T.O., K.L., G.J., and P.B. analyzed data; E.C.L. and V.P. wrote the paper; T.V., T.O., G.J., S.N., P.B., and T.W. provided critical edits to the paper. 


\section{Additional information}

Supplementary Information accompanies this paper at https://doi.org/10.1038/s41467019-11918-y.

Competing interests: J.R.A. and V.P. have applied for a patent related to the treatment of polycystic kidney disease using miR-17 inhibitors. The Patel lab has a sponsored research agreement with Regulus Therapeutics. All authors, apart from A.F., M.Y., and V.P. are current or former employees of, or consultants for, Regulus Therapeutics.

Reprints and permission information is available online at http://npg.nature.com/ reprintsandpermissions/

Peer Review Information Nature Communications thanks the anonymous reviewers for their contribution to the peer review of this work.

Publisher's note Springer Nature remains neutral with regard to jurisdictional claims in published maps and institutional affiliations. (c) (i) Open Access This article is licensed under a Creative Commons Attribution 4.0 International License, which permits use, sharing, adaptation, distribution and reproduction in any medium or format, as long as you give appropriate credit to the original author(s) and the source, provide a link to the Creative Commons license, and indicate if changes were made. The images or other third party material in this article are included in the article's Creative Commons license, unless indicated otherwise in a credit line to the material. If material is not included in the article's Creative Commons license and your intended use is not permitted by statutory regulation or exceeds the permitted use, you will need to obtain permission directly from the copyright holder. To view a copy of this license, visit http://creativecommons.org/ licenses/by/4.0/.

(C) The Author(s) 2019 